\title{
Born to sense: biophysical analyses of the oxygen sensing prolyl hydroxylase from the simplest animal Trichoplax adhaerens
}

This article was published in the following Dove Press journal: Hypoxia

\author{
Kerstin Lippl \\ Anna Boleininger \\ Michael A McDonough \\ Martine I Abboud \\ Hanna Tarhonskaya \\ Rasheduzzaman Chowdhury \\ Christoph Loenarz \\ Christopher J Schofield \\ Chemistry Research Laboratory, \\ University of Oxford, Oxford, UK
}

Correspondence: Christopher J Schofield Chemistry Research Laboratory,

University of Oxford, 12 Mansfield Road,

Oxford, OXI 3TA, UK

Tel +44I865 275625

Fax +44 I865 275674

Email christopher.schofield@chem.ox.ac. uk
Background: In humans and other animals, the chronic hypoxic response is mediated by hypoxia inducible transcription factors (HIFs) which regulate the expression of genes that counteract the effects of limiting oxygen. Prolyl hydroxylases (PHDs) act as hypoxia sensors for the HIF system in organisms ranging from humans to the simplest animal Trichoplax adhaerens.

Methods: We report structural and biochemical studies on the T. adhaerens HIF prolyl hydroxylase (TaPHD) that inform about the evolution of hypoxia sensing in animals.

Results: High resolution crystal structures $(\leq 1.3 \AA)$ of TaPHD, with and without its HIF $\alpha$ substrate, reveal remarkable conservation of key active site elements between $T$. adhaerens and human PHDs, which also manifest in kinetic comparisons.

Conclusion: Conserved structural features of TaPHD and human PHDs include those apparently enabling the slow binding/reaction of oxygen with the active site Fe(II), the formation of a stable 2-oxoglutarate complex, and a stereoelectronically promoted change in conformation of the hydroxylated proline-residue. Comparison of substrate selectivity between the human PHDs and TaPHD provides insights into the selectivity determinants of HIF binding by the PHDs, and into the evolution of the multiple HIFs and PHDs present in higher animals.

Keywords: hypoxia, hypoxic response, oxygen sensing, hypoxia-inducible factor (HIF), evolution, dioxygenase, enzyme structure, PHD/EGLN prolyl hydroxylases, 2-oxoglutarate oxygenase, Trichoplax adhaerens

\section{Introduction}

Hypoxia inducible transcription factors (HIFs) play key roles in maintaining oxygen homeostasis in most, if not all, animals. ${ }^{1-4}$ Extensive work on the HIF mediated response to hypoxia in humans and other higher animals (Figure 1A and B) has revealed that the levels and transcriptional activity of the $\alpha, \beta$-heterodimeric HIF complex are regulated by ferrous iron and 2-oxoglutarate (2OG) dependent oxygenases. ${ }^{4-6}$ Prolyl-4-hydroxylation of the oxygen dependent degradation domains (ODDs) in HIF $\alpha$ is catalyzed by the HIF prolyl hydroxylases (PHDs or EGLNs), and signals for HIF $\alpha$ degradation via the ubiquitin proteasome system. HIF $\alpha$ prolyl hydroxylation strongly promotes its binding to the von-Hippel Lindau protein (pVHL), a targeting component of an E3 ubiquitin ligase complex ${ }^{7-9}$ (Figure 1C). In some animals, a second $2 \mathrm{OG}$ oxygenase, factor inhibiting HIF (FIH), catalyzes asparaginyl-hydroxylation in the $C$-terminal transcriptional activation domain (CTAD) present in both HIF1 $\alpha$ and HIF2 $\alpha$, but 
not in HIF3 $\alpha ;^{10,11}$ this modification inhibits the interaction between HIF and transcriptional coactivators, which are histone acetyl transferases (CBP/p300). ${ }^{11} \mathrm{FIH}$ is active at lower oxygen concentrations than the PHDs ${ }^{12,13}$ and is likely less important from a fundamental hypoxia-sensing perspective.

The PHDs and FIH belong to structurally distinct $2 \mathrm{OG}$ oxygenase subfamilies, with FIH being part of the JumonjiC (JmjC) 2OG oxygenase subfamily IX, ${ }^{14}$ and the PHDs belonging to the $\mathrm{PH}$ (prolyl hydroxylase) subfamily VIII. ${ }^{15}$ However, unlike the HIF $\alpha$-PHD-pVHL "hypoxia sensing triad", the FIH-HIF $\alpha$ CTAD "diad" is not universally conserved, being only sporadically present in simple animals, implying it evolved subsequent to the PHD enabled sensing mechanism. ${ }^{16}$

Extensive genetic and chemical intervention studies support the physiological importance of the HIF $\alpha$-PHDpVHL triad in animals. Indeed, the human PHDs are current therapeutic targets, with PHD inhibitors for the treatment of anemia, via HIF mediated upregulation of erythropoietin, being in late stage clinical trials. ${ }^{17,18}$ In humans, there are three PHDs (PHD1-3) and three HIF $\alpha$ paralogues, but only one FIH. Animal studies imply that PHD2, which, unlike PHD1 and 3, contains a highly conserved Myeloid, Nervy, and DEAF-1 (MYND) finger domain, is the most important of the three mammalian PHDs from a physiological perspective. ${ }^{19,20}$ Unlike humans and other mammals, there is only one PHD present in (most) lower organisms, which is more similar to PHD2 than PHD1 or PHD3. ${ }^{16}$

The human HIF system regulates hundreds of different genes in a context and cell-type dependent manner, with emerging evidence suggesting different roles for HIF $1 \alpha$ and HIF $2 \alpha$ and the three PHDs. ${ }^{12,21-23}$ HIF $1 \alpha$ and HIF $2 \alpha$ each have two ODDs, the $C$-terminal and $N$-terminal (CODD and NODD), respectively, which manifest differential sensitivities to hypoxia. ${ }^{12,24}$ In contrast to the extensive studies conducted on HIF $1 \alpha$ and HIF $2 \alpha$, the biological role of HIF3 $\alpha$, which contains a single ODD, is less understood, with a role for HIF3 $\alpha$ splice variants in the regulation of the hypoxic response being proposed..$^{25-27}$

The three human PHDs also have different ODD selectivities, with PHD1 and PHD2 accepting both NODD and CODD as substrates; however, PHD3 has a strong preference
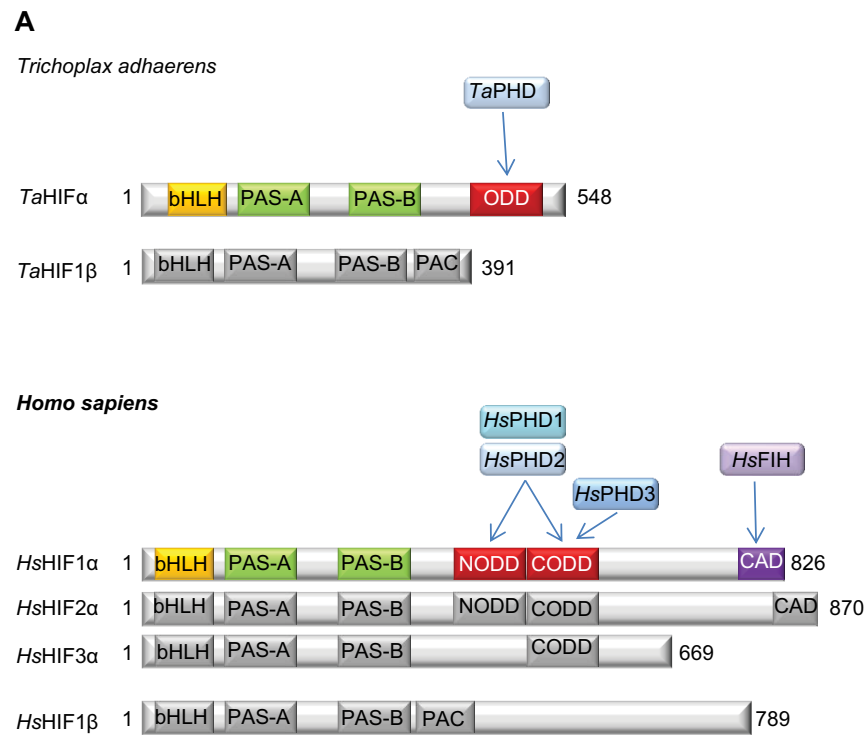

B

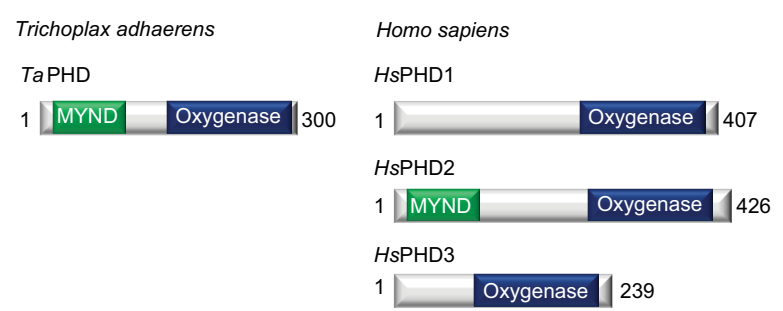

C

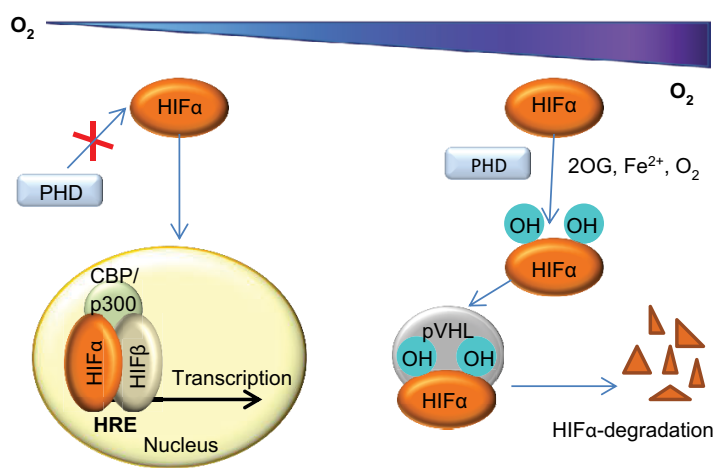

Figure I Overview of the HIF system.

Notes: (A) Comparison of domain architectures of HIF $\alpha$ and HIF $\beta$ in T. adhaerens and humans. In contrast to the multiple PHD/HIF $\alpha$-isoforms and ODDs present in vertebrates, the prime components of the HIF system in T. adhaerens involve only one PHD and one HIF $\alpha$, which has a single ODD. ${ }^{16}$ Arrows indicate assigned prolyl and asparaginyl hydroxylation sites. (B) Domain structures of HIF prolyl hydroxylases (PHDs) in T. adhaerens and humans. Domain acronyms: 2OG dioxygenase domain (oxygenase), MYeloid, Nervy, and DEAF-I (MYND)-type zinc finger domain (MYND). (C) Outline of the conserved mechanism of the response to chronic hypoxia in animals. The HIF transcription factors are regulated by PHD catalyzed hydroxylation of prolyl residues in the ODD(s) of HIF $\alpha$ under normoxia. Recognition of the hydroxylated prolyl residues by $\mathrm{PVHL}$ is followed by ubiquitination by the $\mathrm{E} 3$ ubiquitin ligase, which tags HIF $\alpha$ for proteasomal degradation. In humans, FIH, which is only sporadically present in non-vertebrate animals, ${ }^{16}$ constitutes an additional oxygen dependent regulatory element of HIF activity. FIH catalyzes the hydroxylation of an asparagine residue in the CTAD of $\mathrm{HIF} \alpha$, thus disrupting its interaction with the $\mathrm{CBP} / \mathrm{p} 300$ transcriptional co-activator, and hindering transcriptional activation. ${ }^{\prime \prime}$

Abbreviations: PHD, Prolyl hydroxylases; HIF, hypoxia-inducible transcription factor; ODD, oxygen-dependent degradation domain; bHLH, basic helix-loop-helix motif; PAS, Per-ARNT-Sim domain; CTAD, C-terminal transactivation domain; PAC, PAS-associated C-terminal domain; FIH, factor inhibiting HIF. 
for CODD over NODD as a substrate..$^{21,22}$ Biochemical studies on the PHDs are supportive of their roles as hypoxic sensors. ${ }^{21,28-30}$ An essential requirement for the proposed hypoxia sensing role of the PHDs is that their activity in cells is regulated by oxygen availability. Studies, both in cells and animals, support the proposed hypoxia sensing role of the PHDs, ${ }^{1-9}$ raising the question as to whether their hypoxia sensing role is manifested in specific biochemical properties. There is potential for various factors to help enable PHD activity to be limited by oxygen availability in cells, including regulation of localization, concentrations, or activities of the PHDs and HIF $\alpha$ isoforms. Several studies support the proposal that the kinetic properties of the PHDs are directly related to their sensing role. ${ }^{21,28-30}$ Strikingly, while PHD2, the most conserved of the human PHDs, binds Fe(II) and $2 \mathrm{OG}$ relatively tightly compared to other $2 \mathrm{OG}$ oxygenases, forming an unusually stable enzyme.Fe.2OG complex, the PHD2.Fe.2OG.ODD complex reacts unusually slowly with oxygen (Figure S1). ${ }^{30,31}$ Structural analyses on PHD2 have led to the proposal that its slow reaction with oxygen is related to the active site coordination chemistry, with substitution of an Fe(II) bound water by dioxygen being a limiting step in catalysis. ${ }^{32-36}$ To investigate the proposal that the apparently special kinetic properties of PHDs are a general feature of their hypoxia sensing roles, we have initiated studies on their evolution.

Bioinformatic studies suggest that the HIF $\alpha$-PHD-pVHL triad only exists in animals, though homologues of the PHDs, but not apparently HIF, are present in earlier organisms. ${ }^{16,37,38}$ Roles for PHD homologues in early eukaryotes, ie, hydroxylation of S-phase kinase associated protein 1 (Skp1) in Dictyostelium, ${ }^{37}$ and in some bacteria, ie, hydroxylation of the Elongation Factor EF-Tu ${ }^{38}$ have been identified. However, while these enzymes also catalyze prolyl C-4 hydroxylation and their overall structures are clearly related to the PHDs, the available evidence is that these enzymes differ in their kinetic properties relative to the human PHDs, ${ }^{37-39}$ as is the case for the procollagen prolyl-4-hydroxylases..$^{21,28,40-46}$

The extent to which there is structural conservation of the active sites between the PHDs from different animals has been unclear to date; we have reported experimental evidence for the presence of a functional HIF $\alpha$-PHD-pVHL system in Trichoplax adhaerens, the simplest known animal (Figure S2).${ }^{16}$ Interestingly, the genes encoding for TaHIF $\alpha$ and TaPHD are adjacent in the T. adhaerens genome, suggesting linked evolution, and TaPHD activity can be limited by oxygen availability in human cells. ${ }^{16}$ Here we report crystallographic and biochemical studies on the single PHD present in T. adhaerens (TaPHD, Uniprot ID: I6QVT6), the results of which reveal striking elements of structural and biochemical conservation between TaPHD and human PHD2 (HsPHD2) active sites, supporting oxygen binding/reaction as a key property in the oxygen sensing capability of the PHDs. The results also provide insights into the evolution of ODD binding by PHDs, and help describe how human PHDs achieve ODD selectivity.

\section{Results \\ Steady-state kinetic studies of TaPHD in comparison to HsPHD2}

The HIF system in T. adhaerens is apparently markedly simpler than in humans because it contains only one PHD ( TaPHD), and one HIF $\alpha$ isoform with a single ODD ( $T a$ ODD, Figure 1A). ${ }^{16}$ Although the sequences of human HIF1 $\alpha$ CODD and NODD (HsHIF1 $\alpha$ CODD/NODD), and TaODD differ substantially (Figure 2A), $N$-terminally truncated TaPHD (aa 64-300, $\mathrm{TaPHD}{ }^{64-300}$ ) catalyzes prolyl hydroxylation of both the TaODD 25mer and the human HIF $1 \alpha$ CODD and HIF $1 \alpha$ NODD 19mer substrates, with the latter being hydroxylated less efficiently. ${ }^{16}$ To further investigate the substrate preferences of TaPHD ${ }^{64-300}$, its affinities for TaODD 25mer, human HIF sequences, and its co-substrate (2OG) were determined in steady-state kinetic studies and compared to the truncated human PHD isoform 2 (HsPHD2 ${ }^{181-426}$ ). The kinetic parameters for the catalytic domain of $H s$ PHD $2^{181-426}$ have been shown to be similar to those of the full-length protein. ${ }^{31,59}$

$\mathrm{K}_{\mathrm{m}}$ and $\mathrm{k}_{\text {cat }}$ values (Figure 2B) for HIF peptide substrates and $2 \mathrm{OG}$ were determined under optimized assay conditions described in "Experimental Methods" in Supplementary materials, with the extent of peptide hydroxylation being analyzed by Matrix-Assisted Laser Desorption/Ionization (MALDI) time-of-flight mass spectrometry (MS) (Figures $2 \mathrm{~B}$ and $\underline{\mathrm{S} 3-5}$ ). Consistent with previous reports, ${ }^{16} \mathrm{TaPHD}{ }^{64-300}$ efficiently catalyzes the hydroxylation of its natural substrate TaODD 25mer and the human HIF1 $\alpha$ CODD 19mer peptide. The $H s$ HIF $1 \alpha$ NODD 19 mer, by contrast, is not turned over above background level by $T a \mathrm{PHD}^{64-300}$, at least under the conditions/limits of detection of the assays employed here (Figure $\mathrm{S} 5$ ). TaPHD ${ }^{64-300}$ has a similar affinity to both these substrates, despite their differences in length, as judged by $\mathrm{K}_{\mathrm{m}}$ values $\left(\mathrm{K}_{\mathrm{m}}\left(\right.\right.$ TaODD $\left.25 \mathrm{mer}, T a \mathrm{PHD}^{64-300}\right)=11.5 \pm 1.8 \mu \mathrm{M}$, $\mathrm{K}_{\mathrm{m}}\left(H s \mathrm{HIF} 1 \alpha\right.$ CODD $\left.\left.19 \mathrm{mer}, \mathrm{TaPHD}{ }^{64-300}\right)=15.1 \pm 2.2 \mu \mathrm{M}\right)$. However, the catalytic efficiency of $T a \mathrm{PHD}^{64-300}$ as judged by 
A

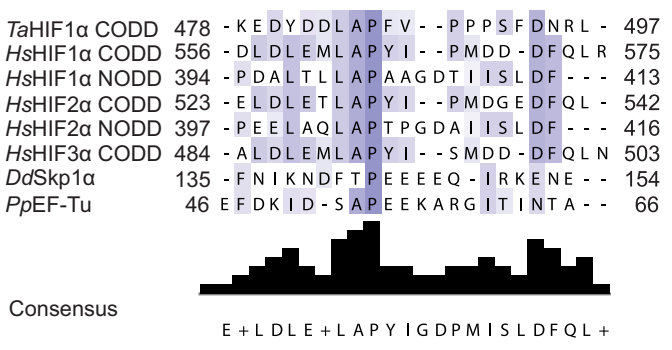

\begin{tabular}{|c|c|c|}
\hline & TaPHD & HsPHD2 \\
\hline $\mathrm{K}_{\mathrm{m}}(\mathrm{TaODD}), \mu \mathrm{M}$ & $11.5 \pm 1.8$ & $40.5 \pm 4.9$ \\
\hline $\mathrm{k}_{\text {cat }}(T a O D D), \mathrm{s}^{-1}$ & $0.026 \pm 0.001$ & $0.035 \pm 0.002$ \\
\hline $\mathrm{K}_{\mathrm{m}}(H s \mathrm{HIF} 1 \alpha \mathrm{CODD}), \mu \mathrm{M}$ & $15.1 \pm 2.2$ & $10.8 \pm 1.9$ \\
\hline $\mathrm{k}_{\mathrm{cat}}(H s \mathrm{HIF} 1 \alpha$ CODD $), \mathrm{s}^{-1}$ & $0.012 \pm 0.001$ & $0.027 \pm 0.001$ \\
\hline $\mathrm{K}_{\mathrm{m}}(2 \mathrm{OG}), \mu \mathrm{M}$ & $1.9 \pm 0.3$ & $15.8 \pm 2.6$ \\
\hline
\end{tabular}

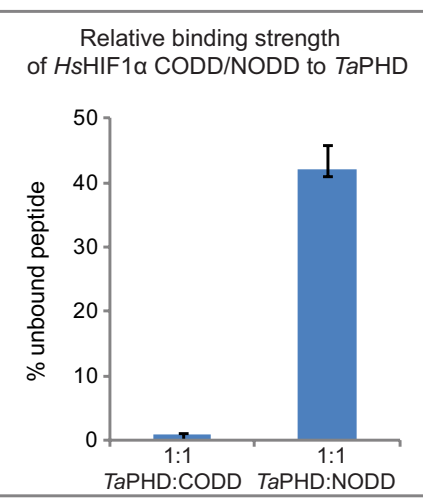

D

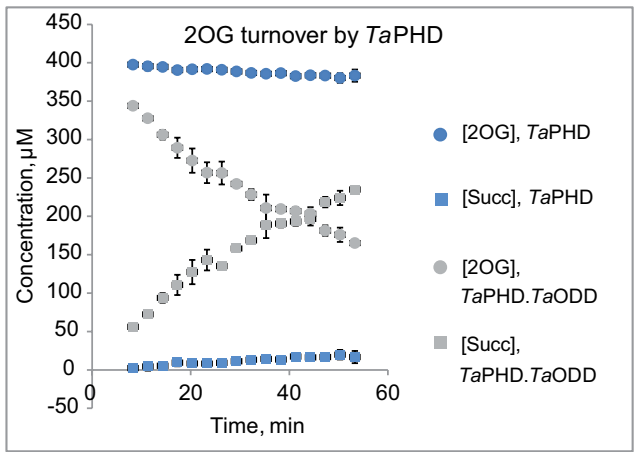

$\mathbf{F}$

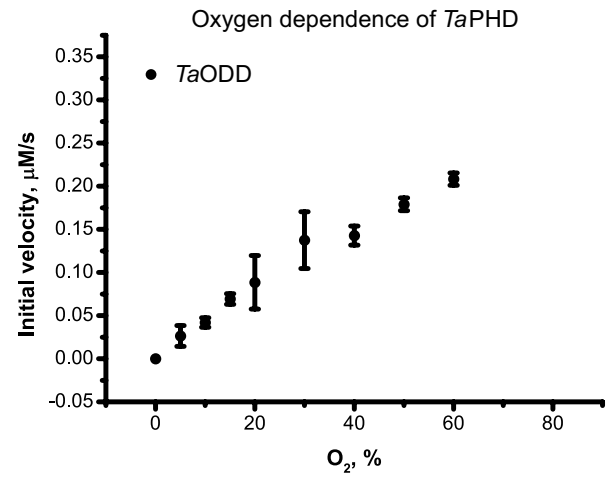

E

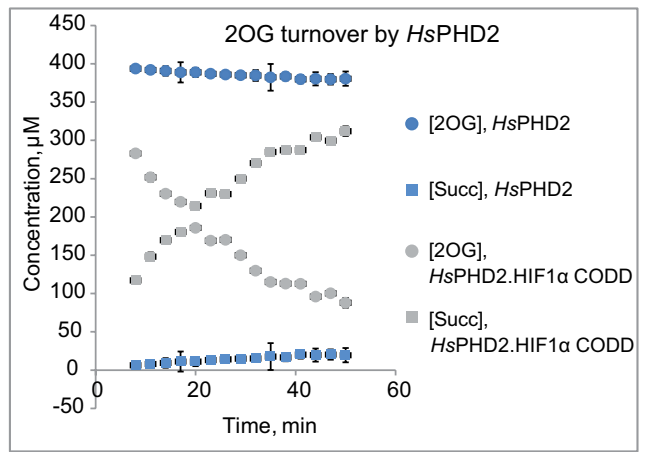

G Oxygen dependence of $\mathrm{HsPHD} 2$

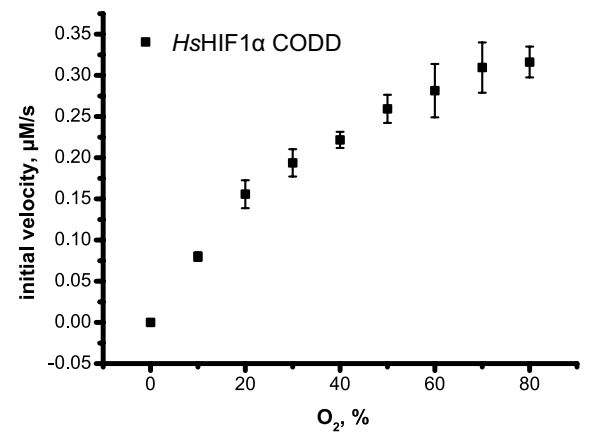

Figure 2 Evidence for conservation of biochemical properties between TaPHD and HsPHD2.

Notes: (A) Sequence alignment of TaHIF $\alpha, H s H I F \alpha$, and substrates of PHD-like enzymes in Dictyostelium discoideum (DdSkpI) and Pseudomonas putida (PpEF-Tu). (B) Kinetic parameters determined for TaPHD and HsPHD2 by MALDI-TOF-MS based assays, conditions: HsPHD2 or TaPHD (3.5 $\mu$ M-7.0 $\mu$ M), HsHIFI $\alpha$ CODD 19mer peptide (DLDLEMLAPYIPMDDDFQL-NH ${ }_{2}, 100 \mu \mathrm{M}$ ) or TaHIF $\alpha$ ODD 25mer peptide (PINEKEDYDDLAPFVPPPSFDNRLY-NH $\left.{ }_{2}, 100 \mu M\right)$, $\left(\mathrm{NH}_{4}\right)_{2} \mathrm{Fe}(\mathrm{II})\left(\mathrm{SO}_{4}\right)_{2}(50 \mu M)$, sodium L-ascorbate $(4 \mathrm{mM})$ and $20 \mathrm{G}$ disodium salt $(300 \mu \mathrm{M})$ in Tris $(50 \mathrm{mM})$, pH 7.5. Initial rates were determined by varying the concentrations of the respective peptide or 2OG. Peptide hydroxylation was analyzed by MALDI-MS; the apparent non-enzymatic Met oxidation was subtracted. Data were fitted with the Michaelis-Menten equation using GraphPad Prism ${ }^{\circledast}$ (errors are indicated as standard deviations, $n=3$ ). (C) Results of ID CLIP HSQC $\left[{ }^{13} \mathrm{C}\right]-H s H I F I \alpha$ CODD and $\left[{ }^{13} \mathrm{C}\right]-H s H I F I \alpha$ NODD displacement experiments with a $\left[{ }^{13} \mathrm{C}\right]$-prolyl-labelled reporter HsHIFI $\alpha$ CODD/NODD peptide reveal a higher binding affinity of TaPHD for HsHIFI $\alpha$ CODD over NODD (errors are indicated as standard deviations, $\mathrm{n}=3$ ); conditions: $\left[{ }^{13} \mathrm{C}\right]$-proline $\mathrm{HsHIFI} \alpha \mathrm{CODD} / \mathrm{NODD}$ (DLDLEMLAPYIPMDDDFQL-NH ${ }_{2} / \mathrm{DALTLLAPAAGDTIISLDF-NH}{ }_{2}, 50 \mu M$ ), TaPHD $(50 \mu \mathrm{M}), 2 \mathrm{OG}$ disodium salt $(50 \mu \mathrm{M})$ buffered with Tris- $\mathrm{D}_{11}(50 \mathrm{mM})$, pH 7.5, in 10\% $\mathrm{D}_{2} \mathrm{O}$ and $90 \% \mathrm{H}_{2} \mathrm{O}$. (D) Comparison of coupled and uncoupled (ie, in the absence of substrate) $2 \mathrm{OG}$ turnover by TaPHD and (E) HsPHD2. 2OG turnover was monitored by 'H CPMG NMR, conditions: TaPHD or HsPHD2 $\left.(20 \mu M),\left(\mathrm{NH}_{4}\right)_{2}\right)$ (III)(SO $\left.)_{4}\right)_{2}(125$ $\mu \mathrm{M})$, sodium L-ascorbate (I mM), HsHIFI $\alpha$ CODD $(500 \mu \mathrm{M})$ or TaHIF $\alpha$ ODD substrate $(500 \mu \mathrm{M})$ (where necessary), and $20 \mathrm{G}$ disodium salt $(400 \mu \mathrm{M})$, in $10 \% \mathrm{D}_{2} \mathrm{O}$ and $90 \%$ $\mathrm{H}_{2} \mathrm{O}$, Tris-D 11 (50 mM), pH 7.5; [Succ]=Succinate. (F) Steady-state $\mathrm{O}_{2}$-dependence of TaPHD and (G) HsPHD2 (published in:29 conditions: $4 \mu \mathrm{M}$ TaPHD/HsPHD2, $100 \mu M$ $\mathrm{TaHIF} \alpha \mathrm{ODD} / \mathrm{HsHIFI} \alpha \mathrm{CODD},\left(\mathrm{NH}_{4}\right)_{2} \mathrm{Fe}(\mathrm{II})\left(\mathrm{SO}_{4}\right)_{2}(50 \mu \mathrm{M}), 2 \mathrm{OG}$ disodium salt $(300 \mu \mathrm{M})$ and sodium L-ascorbate $(4 \mathrm{mM})$ in Tris $(50 \mathrm{mM})$, pH 7.5 were incubated at $37^{\circ} \mathrm{C}$ under different $\% \mathrm{O}_{2}$. The extent of hydroxylation was analyzed by MALDI-ToF-MS (errors are indicated as standard deviations, $\mathrm{n}=3$ ). 
$\mathrm{k}_{\text {cat }}$ is lower with the human CODD sequence $\left(\mathrm{k}_{\text {cat }}(H s \mathrm{HIF} 1 \alpha\right.$ CODD 19mer, $\left.T a \mathrm{PHD}^{64-300}\right)=0.012 \pm 0.001 \mathrm{~s}^{-1}, \mathrm{k}_{\text {cat }}(T a \mathrm{ODD}$ $\left.\left.25 \mathrm{mer}, \mathrm{TaPHD}{ }^{64-300}\right)=0.026 \pm 0.001 \mathrm{~s}^{-1}\right)$. HsPHD2 ${ }^{181-426}$, by comparison, manifests a decreased affinity for the $\mathrm{TaODD}$ $\left(\mathrm{K}_{\mathrm{m}}\left(H s \mathrm{HIF} 1 \alpha\right.\right.$ CODD 19mer, $\left.H s \mathrm{PHD} 2^{181-426}\right)=10.8 \pm 1.9 \mu \mathrm{M}$, $\left.\mathrm{K}_{\mathrm{m}}\left(T a \mathrm{ODD}, H s \mathrm{PHD} 2^{181-426}\right)=40.5 \pm 4.9 \mu \mathrm{M}\right) . H s \mathrm{PHD} 2^{181-426}$, however, catalyzes hydroxylation of $H s \mathrm{HIF} 1 \alpha$ CODD $19 \mathrm{mer}$ and TaODD 25mer with comparable efficiency $\mathrm{k}_{\text {cat }}(H s \mathrm{HIF} 1 \alpha$ CODD 19mer, $H s$ PHD $\left.2^{181-426}\right)=0.027 \pm 0.001 \mathrm{~s}^{-1}, \mathrm{k}_{\text {cat }}(T a O D D$ $\left.\left.25 \mathrm{mer}, H s \mathrm{PHD} 2^{181-426}\right)=0.035 \pm 0.002 \mathrm{~s}^{-1}\right)$.

Notably, the affinity of TaPHD ${ }^{64-300}$ for $2 \mathrm{OG}\left(\mathrm{K}_{\mathrm{m}}(2 \mathrm{OG}\right.$, $\left.T a \mathrm{PHD}^{64-300}\right)=1.9 \pm 0.3 \mu \mathrm{M}$ ) is higher than for $H_{s} \mathrm{PHD}^{181-426}$ $\left(\mathrm{K}_{\mathrm{m}}\left(2 \mathrm{OG}, H_{s} \mathrm{PHD} 2^{181-426}\right)=15.8 \pm 2.6 \mu \mathrm{M}\right)$, as judged by $\mathrm{K}_{\mathrm{m}}$ comparisons. The higher affinity of $2 \mathrm{OG}$ for $\mathrm{TaPHD}{ }^{64-300}$ is in accord with the observation of formation of a stable $\mathrm{TaPHD}$. Fe.2OG complex, with a half-life $>24 \mathrm{~h} .{ }^{16}$ The kinetic parameters reported here for the affinity of $H s \mathrm{HIF} 1 \alpha$ CODD $19 \mathrm{mer}$ and $2 \mathrm{OG}$ for $H s \mathrm{PHD} 2^{181-426}$ are consistent with previous studies, ${ }^{29} \mathrm{~K}_{\mathrm{m}}\left(H s \mathrm{HIF} 1 \alpha\right.$ CODD $\left.19 \mathrm{mer}, H s \mathrm{PHD} 2^{181-426}\right)=9 \pm 3$ $\left.\mu \mathrm{M}, \mathrm{K}_{\mathrm{m}}\left(2 \mathrm{OG}, H s \mathrm{PHD} 2^{181-426}\right)=13 \pm 2 \mu \mathrm{M}\right)$.

\section{TaPHD binds $H s H I F I \alpha$ NODD less efficiently than HsHIFI $\alpha$ CODD}

The observed preference of $T a \mathrm{PHD}^{64-300}$ for $H s \mathrm{HIF} 1 \alpha$ CODD over $H s H I F 1 \alpha$ NODD is in agreement with the finding of one ODD in the genome of T. adhaerens, which is closer in sequence to the majority of CODD, compared to NODD sequences (Figure 2A). ${ }^{16}$ While a NODD-like sequence is present in all vertebrates, NODD is apparently not present in some non-vertebrates, eg, Placozoa and Cnidaria, suggesting that the CODD sequence might have evolved prior to that of NODD. ${ }^{16}$ Notably, like TaPHD ${ }^{64-300}$, one of the human PHD isoforms, $H s \mathrm{PHD} 3$, is highly selective for $H s \mathrm{HIF} 1 \alpha / 2 \alpha$ CODD over $H s$ HIF $1 \alpha / 2 \alpha$ NODD. ${ }^{1,21,47}$

To more directly investigate the extent to which $T a \mathrm{PHD}^{64}$ ${ }^{300}$ can bind $H s$ HIF $1 \alpha$ NODD and CODD, we performed 1D CLIP HSQC NMR binding studies with $H s$ HIF $1 \alpha$ CODD and $H s \mathrm{HIF} 1 \alpha$ NODD peptides labeled with $\left[{ }^{13} \mathrm{C}\right]$ at the target prolyl residues which undergo hydroxylation during catalysis (Figure 2C). The $\left[{ }^{13} \mathrm{C}\right]$-labeled "reporter" $H s \mathrm{HIF} 1 \alpha \mathrm{CODD} /$ NODD peptides were incubated with $\mathrm{Zn}(\mathrm{II})$ and $2 \mathrm{OG}$ in the presence and absence of $T a$ PHD $^{64-300}$ (1:1 enzyme:ODD, for conditions see "Experimental methods" in Supplementary materials). The decrease of the reporter signal upon binding to the enzyme was measured to investigate the relative binding strength (Figure 2C). Consistent with the kinetic studies, the NMR results reveal that $T a \mathrm{PHD}^{64-300}$ strongly discriminates between binding of $H_{s} \mathrm{HIF} 1 \alpha$ CODD and $H_{s} \mathrm{HIF} 1 \alpha$ NODD, as $\sim 40 \%$ of the $H s H I F 1 \alpha$ NODD peptide remained unbound, while $H s H I F 1 \alpha$ CODD was apparently completely bound to $T a \mathrm{PHD}^{64-300}$, showing that $T a \mathrm{PHD}^{64-300}$ binds $H s \mathrm{HIF} 1 \alpha \mathrm{CODD}$ better than $H s$ HIF $1 \alpha$ NODD.

\section{Monitoring the coupled and uncoupled 2OG turnover of TaPHD}

$2 \mathrm{OG}$ dependent oxygenases catalyze the uncoupled turnover of $2 \mathrm{OG}$ to succinate and $\mathrm{CO}_{2}$ in the absence of their prime substrate to varying extents; ${ }^{48}$ in the case of $H s$ PHD2, it has been shown that $20 \mathrm{G}$ decarboxylation is strongly coupled to CODD hydroxylation..$^{31}$ To directly compare the extent of uncoupled turnover in $\mathrm{TaPHD}{ }^{64-300}$ and $H s \mathrm{PHD} 2^{181-426}$, succinate formation and $2 \mathrm{OG}$ depletion were monitored by ${ }^{1} \mathrm{H}-\mathrm{NMR}^{49}$ in the presence and absence of their respective natural substrates (Figure 2D and E). The results demonstrate that the rate of uncoupled 2OG turnover is very low for both $\mathrm{TaPHD}^{64-300}$ and HsPHD2 ${ }^{181-426}$; $2 \mathrm{OG}$ decarboxylation is strongly increased in presence of substrate, implying conservation of (at least some) kinetic properties between $H_{s}$ PHD2 and TaPHD. Overall, these results support the proposal that the strong binding of $\mathrm{Fe}$ (II) and $2 \mathrm{OG}$, and the formation of a stable $H s$ PHD2.Fe.2OG complex in the absence of $H s H I F \alpha$, are conserved properties of the PHDs.

\section{Oxygen dependence of TaPHD}

In order for the PHDs to act as hypoxia sensors in cells, their activity must be limited by oxygen availability. This role is proposed to be reflected in the slow reaction of (at least) the $H s$ PHD2.CODD/NODD.Fe.2OG complexes with oxygen..$^{29,31}$ It is also proposed to manifest in the unusually high $\mathrm{K}_{\mathrm{m}}$ value (for $2 \mathrm{OG}$ oxygenases) of $H s \mathrm{PHD} 2$ for oxygen $\left(\mathrm{K}_{\mathrm{m}}\left(\mathrm{O}_{2}\right.\right.$, $H_{s}$ PHD2) $\left.>400 \mu \mathrm{M}\right) .{ }^{21,28,29,50}$ In order to study the $\mathrm{O}_{2}$ dependence of the TaPHD-catalyzed reaction, steady-state kinetics, using reported experimental conditions for $H s \mathrm{PHD} 2 ;{ }^{29}$ were conducted. $T a \mathrm{PHD}^{64-300}$ was reacted with its peptidic substrate in sealed glass-vials under varied oxygen concentrations, ${ }^{29}$ the initial rates of $T a$ ODD 25 mer hydroxylation were determined by MALDI-TOF-MS (Figure 2F and G). No saturation of $\mathrm{O}_{2}$ was reached for the $T a \mathrm{PHD}^{64-300}$-catalyzed reaction with $\mathrm{TaODD} 25 \mathrm{mer}$ at $60 \% \mathrm{O}_{2}$ level, resulting in a $\mathrm{K}_{\mathrm{m}}\left(\mathrm{O}_{2}\right.$, $\left.T a \mathrm{PHD}^{64-300}\right)>400 \mu \mathrm{M}$. This result suggests that $\mathrm{TaPHD}$ has the potential to react to small changes in atmospheric $\mathrm{O}_{2}$ partial pressure and could act as an effective oxygen sensor, indicating that the core biochemical features of $H_{s} \mathrm{PHD} 2$ in the human oxygen sensing machinery may well be conserved in $T a \mathrm{PHD}$. It has been reported that $H s \mathrm{PHD} 2$ reacts relatively slowly with oxygen, compared to other $2 \mathrm{OG}$ oxygenases as 
determined by stopped flow/UV-visible spectroscopy experiments. ${ }^{29,31}$ It was not possible to conduct a similar study on TaPHD for practical reasons (ie, neither TaPHD ${ }^{64-300}$, nor other tested constructs $T a \mathrm{PHD}^{1-300}$ and $T a \mathrm{PHD}^{79-300}$ could reach a sufficiently high protein concentration necessary for stopped-flow assays due to aggregation).

\section{Crystallization of $\mathrm{N}$-terminally truncated T. adhaerens PHD (TaPHD ${ }^{64-300}$ )}

In order to investigate the extent to which the structural features proposed to be necessary for oxygen sensing are conserved between $H s$ PHD2 and TaPHD, we attempted to obtain a crystal structure of TaPHD in complex with $\mathrm{Mn}(\mathrm{II})$, a non-reactive substitute for Fe(II), using a TaPHD construct (aa 64-300, TaPHD ${ }^{64-300}, \mathrm{MW}=29.2 \mathrm{kDa}$ ) lacking the $N$-terminal MYND domain, which could be produced efficiently in Escherichia coli. The shorter construct (TaPHD ${ }^{64-300}$ ) yielded crystals that diffracted to $1.2 \AA$ resolution (Table 1). The structure was solved by molecular replacement, using $H_{S}$ PHD2 (PDB: 2G19), ${ }^{32}$ as a search model. To gain insight into the substrate-binding mode of TaPHD, we then co-crystallized TaPHD ${ }^{64-300}$ with a 21 mer peptide fragment of its substrate, the TaHIF $\alpha$ ODD ( $T a O D D$, residues E477-L497), $\mathrm{Mn}(\mathrm{II})$ and $N$-oxalylglycine (NOG), a close $2 \mathrm{OG}$ analog. The TaPHD.TaODD.Mn.NOG structure (Table 1) was solved to a resolution of $1.3 \AA$ by molecular replacement, using the structure without $\mathrm{TaODD}$ as a search model. The substrate-bound and unbound forms of TaPHD crystallized in the $P 1$ and $P 2_{1}$ space groups, respectively, reflecting different packing, possibly relating to structural changes in TaPHD induced upon substrate binding.

Table I Data collection and refinement statistics

\begin{tabular}{|c|c|c|}
\hline & $\begin{array}{l}\text { TaPHD.Mn } \\
\text { (PDB: 6EYI) }\end{array}$ & $\begin{array}{l}\text { TaPHD.TaODD } \\
\text { (PDB: 6FOW) }\end{array}$ \\
\hline Wavelength $(\AA)$ & 0.97930 & 0.97950 \\
\hline Resolution range $(\AA)^{\mathrm{a}}$ & $38.39-1.20(1.24-1.20)$ & $30.49-1.30(1.35-1.30)$ \\
\hline Space group & $P 2_{1}$ & PI \\
\hline Unit cell (a b c, $\alpha \beta \gamma)$ & $40.5859 .4 \mid 51.20,90$ I00.74 90 & 40.844 I.32 42.08, II 4.0695 .67 I03.69 \\
\hline Total reflections & 640,269 & 377,792 \\
\hline Unique reflections ${ }^{\mathrm{a}}$ & 74,236 (6949) & $55,424(5243)$ \\
\hline Multiplicity & $8.6(4.9)$ & $6.8(4.1)$ \\
\hline Completeness (\%) ${ }^{\mathrm{a}}$ & 99.24 (93.39) & $94.83(89.72)$ \\
\hline Mean $I / \sigma(l)^{\mathrm{a}}$ & $25.9(2.0)$ & $14.5(2.6)$ \\
\hline Wilson B-factor & 13.06 & 13.89 \\
\hline$R_{\text {merge }} \mathrm{b}$ & 0.084 & 0.112 \\
\hline Reflections used in refinement ${ }^{\mathrm{a}}$ & 74,191 (6940) & $55,4 \mid 3(5244)$ \\
\hline Reflections used for R-free ${ }^{a}$ & $3,738(333)$ & $2,775(264)$ \\
\hline$R_{\text {work }}^{c, a}$ & $0.1331(0.2088)$ & $0.1475(0.2140)$ \\
\hline$R_{\text {free }}^{d, a}$ & $0.1483(0.2396)$ & $0.1687(0.2430)$ \\
\hline Number of non-hydrogen atoms & 2,026 & 2,146 \\
\hline Macromolecules & 1,808 & 1,933 \\
\hline Ligands & 19 & II \\
\hline Solvent & 199 & 202 \\
\hline Protein residues & 211 & 239 \\
\hline RMS (bonds) $(\AA)^{\mathrm{e}}$ & 0.011 & 0.012 \\
\hline RMS (angles) $\left({ }^{\circ}\right)^{e}$ & 1.09 & 1.09 \\
\hline Ramachandran favored (\%) & 97.56 & 98.24 \\
\hline Ramachandran allowed (\%) & 2.44 & 1.76 \\
\hline Ramachandran outliers (\%) & 0.00 & 0.00 \\
\hline Rotamer outliers (\%) & 0.98 & 0.48 \\
\hline Clashscore & 1.37 & 2.36 \\
\hline Average B-factor $\left(\AA^{2}\right)$ & 22.75 & 20.67 \\
\hline Macromolecules $\left(\AA^{2}\right)$ & 21.24 & 19.36 \\
\hline Ligands $\left(\AA^{2}\right)$ & 31.98 & 12.99 \\
\hline Solvent $\left(\AA^{2}\right)$ & 35.59 & 33.67 \\
\hline
\end{tabular}

Notes: aParentheses indicate high resolution shell. ${ }^{b} R_{\text {merge }}=\sum_{j} \sum_{h}\left|I_{h j}-<I_{h}>\right| / \sum_{j} \sum_{h}<I_{h}>\times 100 .{ }^{c} R_{\text {work }}=\sum||$ Fobs $|-| F c a l c|| / F$ Fobs $|\times| 00$. ${ }^{d} R_{\text {free' }}$, based on $2 \%-5 \%$ of the total

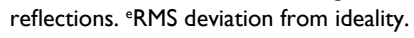




\section{Overall structure of TaPHD}

The structure of $T a$ PHD (Figure 3A) contains the conserved double-stranded $\beta$-helix core-fold (DSBH, or "jelly roll"motif), which is present in all characterized 2OG dependent oxygenases, including the human PHDs. ${ }^{15}$ The DSBH is comprised of the major ( $\beta \mathrm{I}, \beta \mathrm{VIII}, \beta \mathrm{III}, \beta \mathrm{VI})$ and minor ( $\beta \mathrm{II}, \beta \mathrm{VII}, \beta \mathrm{IV}, \beta \mathrm{V}$ ) $\beta$-sheets between which the metal and $2 \mathrm{OG}$ binding sites are sandwiched. As with $H_{s} \mathrm{PHD} 2$, the major $\beta$-sheet core is stabilized by three $\alpha$-helices $(\alpha 1-3)$. The overall folds of the substrate-unbound TaPHD (TaPHD. $\mathrm{Mn}$ ) and the substrate-bound TaPHD.TaODD complex are very similar (Figure $3 \mathrm{~A}$ and $\mathrm{B}, \mathrm{RMSD}$ for all $\mathrm{C} \alpha=0.15 \AA$ ). Eighteen residues of the 21 mer $T a \mathrm{ODD}$ peptide (A480 ${ }_{T a \mathrm{ODD}}{ }^{-}$ $\mathrm{L}_{497_{T a \mathrm{ODD}}}$, Figure 3B) are visible in the TaPHD.TaODD structure.

Clear differences between the TaPHD.Mn and TaPHD. $T a$ ODD structures manifest in the mobile " $\beta 2 / \beta 3$-fingerloop", ${ }^{32-34}$ which is disordered in the substrate-unbound TaPHD structure (missing residues Q137 ${ }_{T a \mathrm{PHD}}-\mathrm{R} 146_{\text {TaPHD }}$ ). In the substrate-bound structure, however, the $\beta 2 / \beta 3$-finger-loop is ordered and makes extensive contacts with the substrate. By contrast, the $\beta 7(\mathrm{VI}) / \beta 8(\mathrm{~V})$-loop residue $\mathrm{N} 245_{\text {TaPHD }}$ is not observed in the TaPHD.TaODD structure, unlike in the substrate-unbound TaPHD.Mn structure, in which the electron density of the complete $\beta 7(\mathrm{VI}) / \beta 8(\mathrm{~V})$-loop is apparent (Figures 3-6, S4 and S6).

\section{Active site of TaPHD}

The active site pocket of TaPHD, positioned at one end of the DSBH, deeply embeds the metal ion (Mn, substituting for $\mathrm{Fe}$ ) and the co-substrate (NOG, substituting for 2OG, Figure $3 \mathrm{~B}$ and $\mathrm{C}$ ). The highly buried nature of the $2 \mathrm{OG}$ binding site likely contributes to the formation of a stable $T a$ PHD.Fe.2OG complex, as reflected in the observed low rate of uncoupled $2 \mathrm{OG}$ turnover by TaPHD (Figures 2D and $3 \mathrm{~B}$ ).

In the TaPHD.Mn structure without $T a \mathrm{ODD}$, the manganese ion is octahedrally coordinated by a conserved triad of residues (H209 ${ }_{T a \mathrm{PHD}}, \mathrm{D} 211_{T a \mathrm{PHD}}$, and $\mathrm{H} 270_{T a \mathrm{PHD}}$ ), an acetate ion, and two water molecules. ${ }^{48,51}$ Deep within the $20 \mathrm{OG}$ binding pocket an additional acetate ion forms a salt bridge with R279 $T a \mathrm{PHD}$, apparently mimicking $2 \mathrm{OG} \mathrm{C} 5$ carboxylate binding (Figure 3A).

The 6-coordinate state of the metal is retained in the TaPHD.TaODD complex. Metal-ion binding involves the highly conserved triad of metal-coordinating residues, and an ordered, metal-bound water molecule (W1) that is, in addition to the metal coordination, stabilized via hydrogen bonding with the metal-ligating side chain of D211 $1_{\text {TaPHD }}$ (Figures $3 \mathrm{C}$ and 6). Electron density corresponding to the $2 \mathrm{OG}$ analog inhibitor, NOG, is clearly visible in the TaPHD. $T a$ ODD complex structure. NOG coordinates to the metal ion in a bidentate manner and interacts with R279 ${ }_{T a \mathrm{PHD}}$ by
A

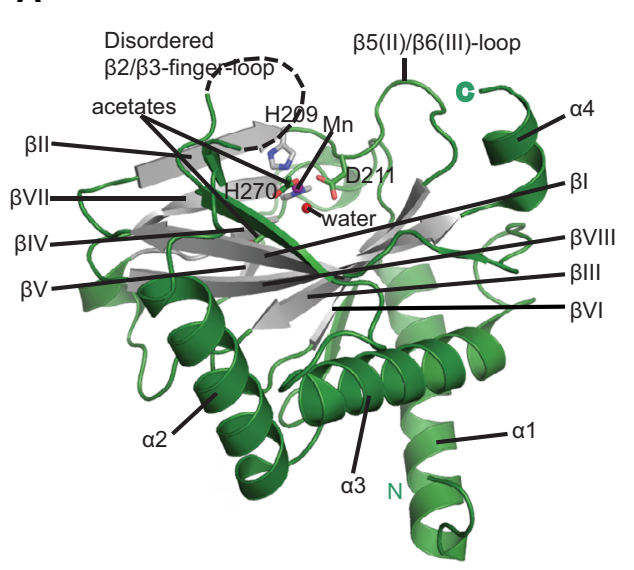

TaPHD.Mn
B

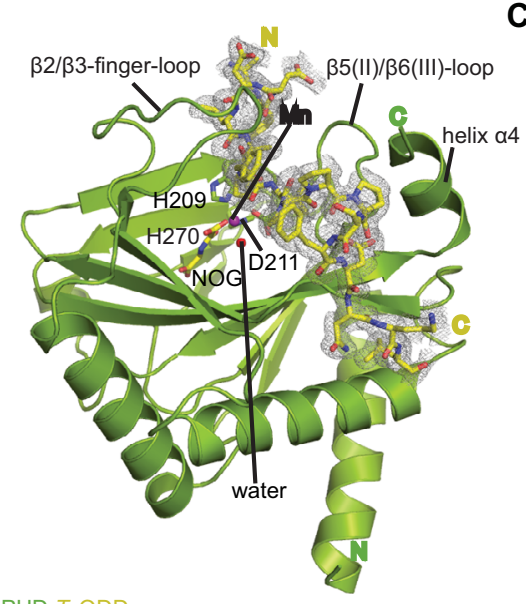

TaPHD. TaODD
C

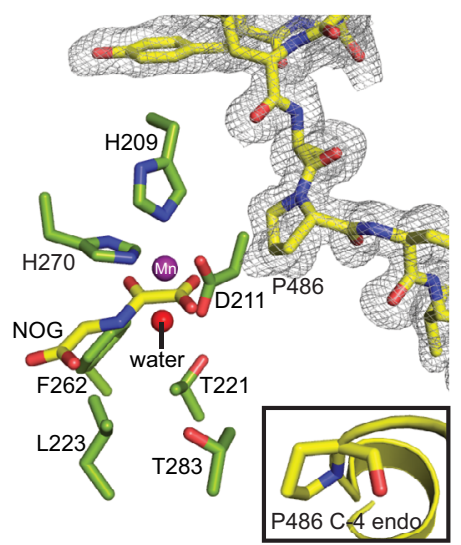

TaPHD.TaODD

Figure 3 Views from crystal structures of the Trichoplax adhaerens prolyl hydroxylases (PHD) without substrate bound (TaPHD) and in complex with a fragment of its substrate (TaPHD.TaODD).

Notes: (A) Secondary structural elements in TaPHD comprise four $\alpha$ helices and ten $\beta$ strands, eight of which form the double-stranded $\beta$-helix core fold (DSBH, gray, Roman numerals I-VIII). (B) Overall binding mode of TaODD to TaPHD in the TaPHD.TaODD complex structure showing the $2 \mathrm{~F}_{\mathrm{o}}-\mathrm{F}_{\mathrm{c}}$ electron density map for the peptidic substrate (gray mesh, contoured to $1.0 \sigma$ ). (C) Active site close-up of TaPHD.TaODD reveals that the P486 TaODD C-4 methylene adopts an endo-conformation (2F$\mathrm{F}_{\mathrm{c}}$ density, gray mesh, contoured to $\mathrm{I} .0 \mathrm{\sigma}$ ). The metal ion (manganese substituting for iron, purple sphere) is octahedrally coordinated by a triad of residues ( $\mathrm{H} 209_{\text {ToPHD' }}$, $\mathrm{D} 2 \mathrm{II}_{\mathrm{TaPHD}}$, and $\mathrm{H} 27 \mathrm{~T}_{\mathrm{TaPHD}}$ ), $\mathrm{N}$-oxalylglycine (NOG), and a water molecule (WI, red sphere). The stable metal-water coordination observed here is conserved in $\mathrm{HsPHD2,32,34}$ where it is proposed to enable the oxygen sensing ability in HsPHD2. 
A

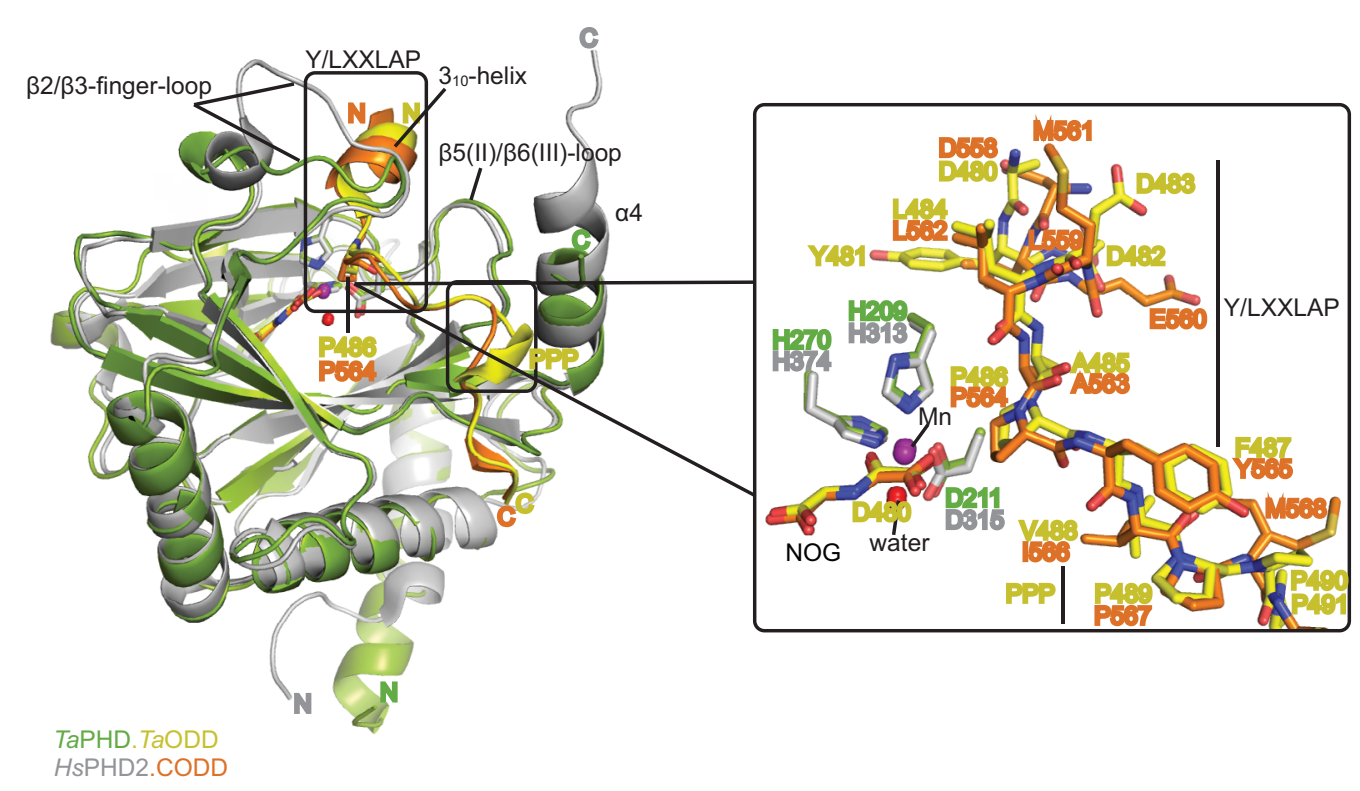

B

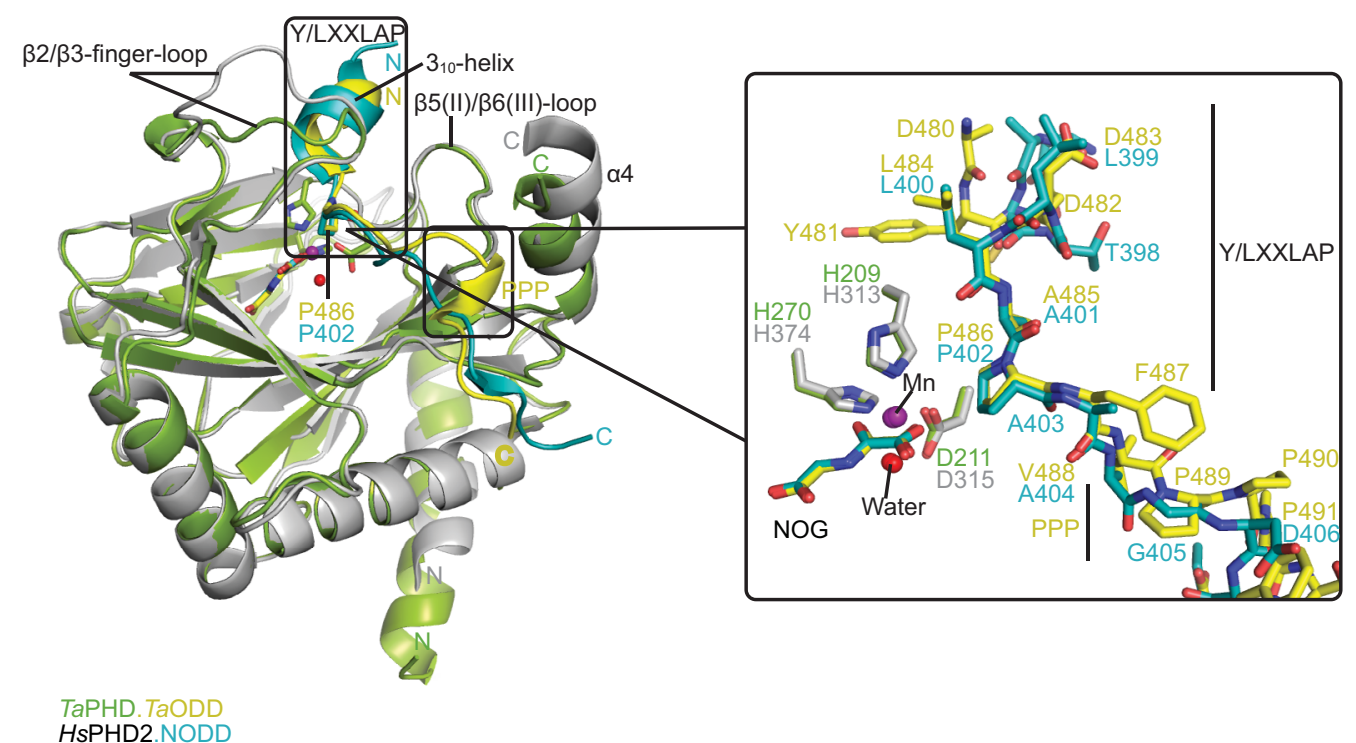

Figure 4 Comparison of ODD binding modes by the T. adhaerens and human HIF $\alpha$ PHD for the TaPHD.TaODD, HsPHD2.CODD and HsPHD2.NODD substrate structures.

Notes: (A) Superimposition of structural views of TaPHD.TaODD with HsPHD2.CODD (PDB: 3HQR) and (B) TaPHD.TaODD with HsPHD2.NODD (PDB: 5L9V) reveals major differences between the structures in the PHD flexible $\beta 2 / \beta 3$ finger-loop and PHD C-terminal substrate binding interfaces. Notably, the Pro-Pro-Pro motif in TaODD (PPP motif, P489-49I ${ }_{\text {ToODD }}$ ) adopts a helical bend, which aligns poorly with $H s H I F I \alpha$ CODD and, particularly, with HsHIFI $\alpha$ NODD.

Abbreviations: $T$. adhaerens, Trichoplax adhaerens; ODD, oxygen dependent degradation domain.

its $\mathrm{C} 5$ carboxylate. The 1-carboxylate of NOG is positioned at the coordination site closest to the hydroxylation target, $\mathrm{P}^{4} 86_{T a \mathrm{ODD}}$, making the remaining metal coordination site, occupied by water $\mathrm{W} 1$, less accessible, and thus apparently hindering oxygen binding to the metal ion (Figure 6)..$^{33,34}$

Crystal structures of the human PHD isoform 2 ( $H s$ PHD2) in complex with its substrates $H s \mathrm{HIF} 1 \alpha$ CODD (referred to as $H s$ PHD2.CODD, PDB: $3 \mathrm{HQR}^{34}$ ) and $H s \mathrm{HIF} 1 \alpha$
NODD (referred to as HsPHD2.NODD, PDB: 5L9V ${ }^{33}$ ) have been reported (Figure 4). The overall active site arrangement observed in the TaPHD.TaODD complex is very similar to those observed in the HsPHD2.CODD/NODD complexes (Figures 4-6, ${ }^{33,34}$ ). Such conservation includes the octahedral coordination of the metal ion by $\mathrm{H} 313_{\mathrm{HsPHD} 2}, \mathrm{D} 315_{\mathrm{Hs} \mathrm{PHD} 2}$, and $\mathrm{H} 374_{H S \mathrm{PHD}}$, NOG, and a metal-bound water molecule. ${ }^{33,34,52}$ The observation of a well-ordered metal bound water in both 
A

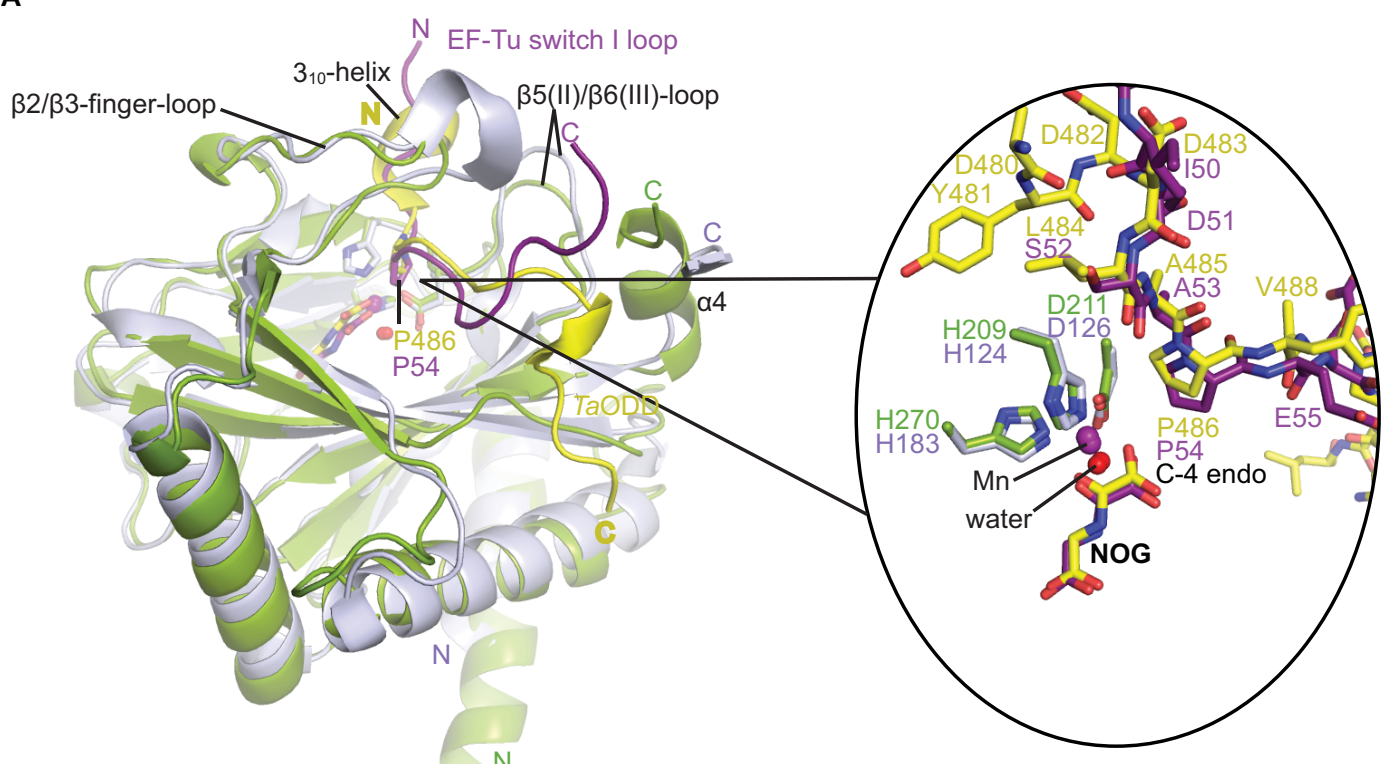

TaPHD. TaODD

PPHD.EF-Tu

\section{B}

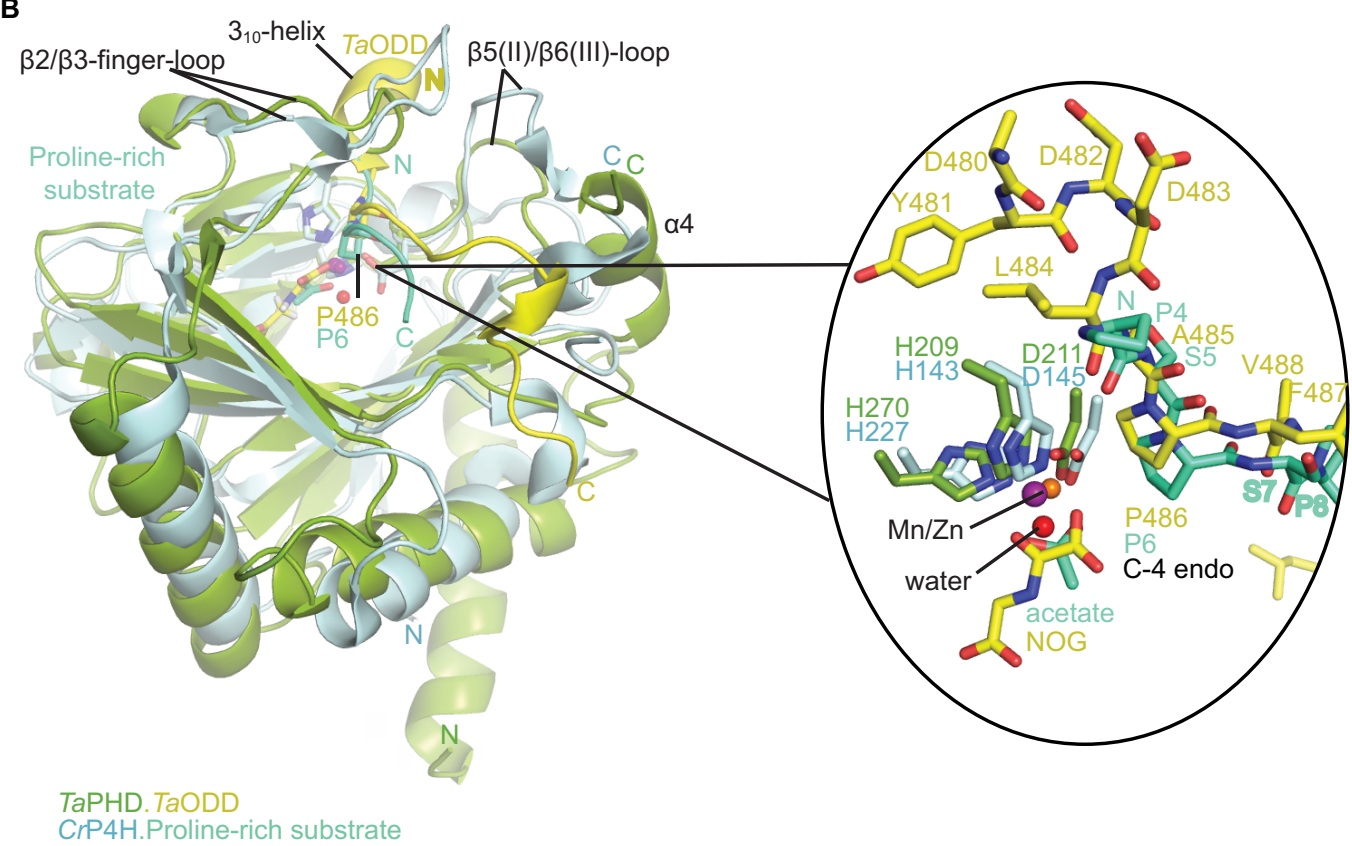

Figure 5 Comparison of substrate binding modes by TaPHD, CrP4H and Pseudomonas putida PPHD.

Notes: Overall superimposition and active site close-up of the TaPHD.TaODD complex with (A) P. putida PPHD (PDB: 4IW3) and (B) CrP4H (PDB: 3GZE) in complex with a proline rich peptidic substrate reveals the conservation of the substrate-binding mode involving the conformationally flexbile $\beta 2 / \beta 3$-finger-loop (in all cases) and the C-terminal $\alpha 4$-helix (in case of TaPHD and PPHD).

TaPHD and HsPHD2 structures is important from a hypoxia sensing perspective, because displacement of this water from the metal is required for oxygen to bind, and is proposed to be rate limiting in $H s$ PHD2 catalysis. ${ }^{32,34,53}$ The buried nature of the metal, the $2 \mathrm{OG}$ analog NOG, and the associated ligating water molecule, as observed both in TaPHD and HsPHD2, are likely substantially responsible for the unusual stability of the HsPHD2.Fe.2OG complex, ${ }^{30}$ compared to other $2 \mathrm{OG}$ oxygenases such as FIH, which is active at lower oxygen concentrations. ${ }^{12,13,54}$

Taken together, the observation of conserved active site arrangements in the TaPHD.TaODD and HsPHD2.CODD crystal structures correlates well with the steady-state kinetic parameters determined for $20 \mathrm{O}$ and oxygen, imply- 
A

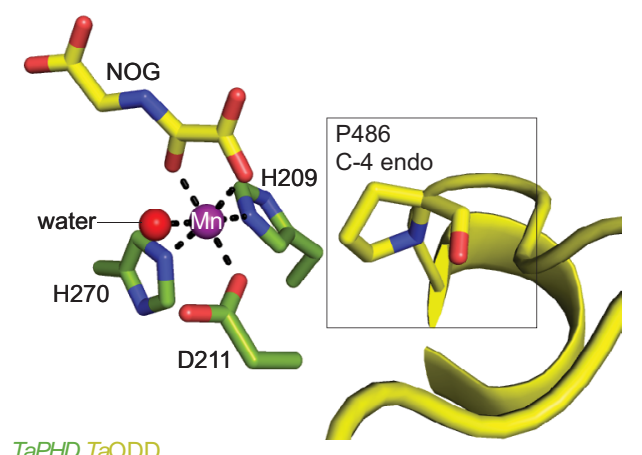

TaPHD. TaODD

C

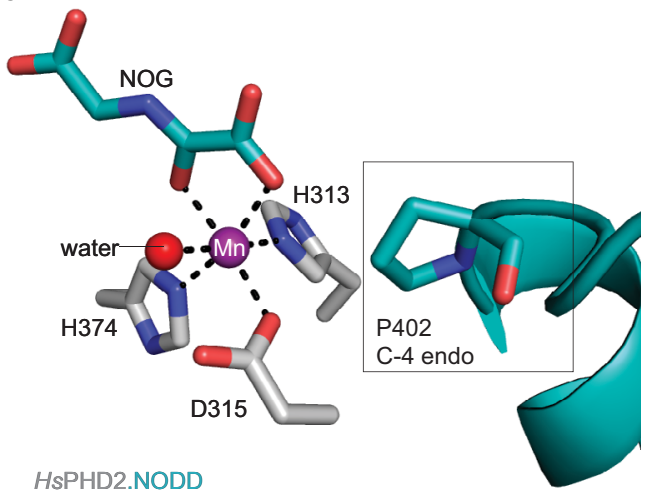

E

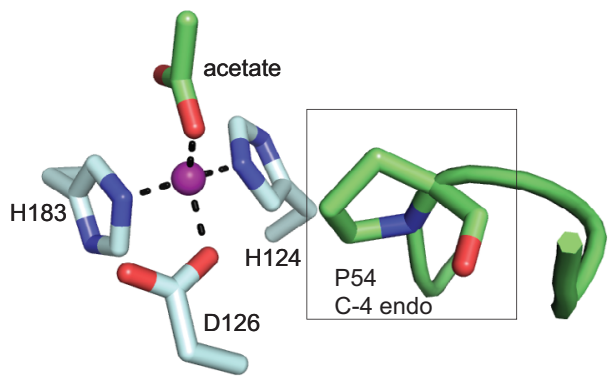

CrP4H.Proline-rich substrate
B

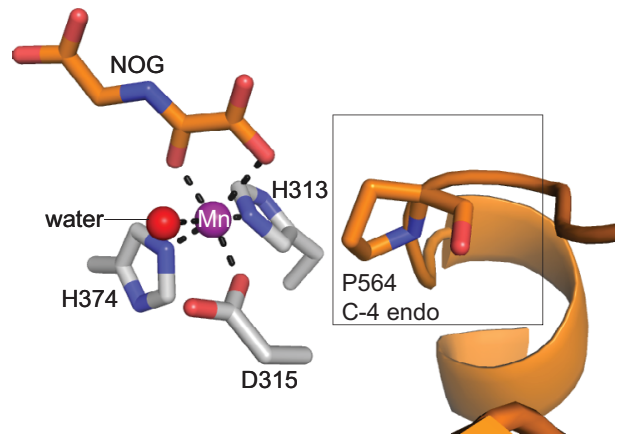

HsPHD2.CODD

D

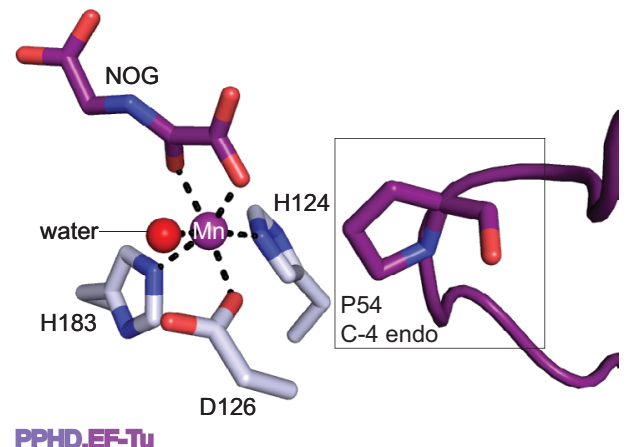

$\mathbf{F}$

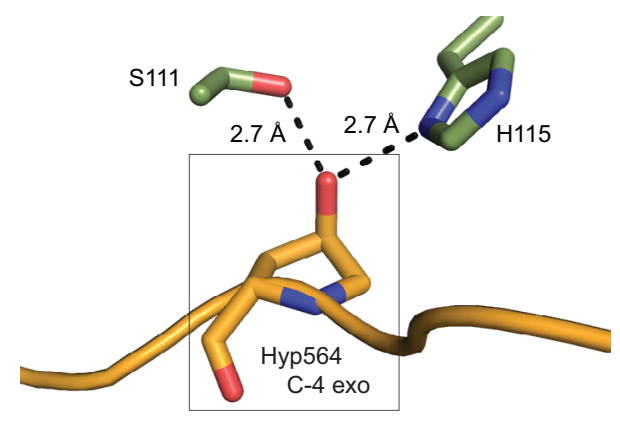

VCB.CODD

Figure 6 Active site metal region details and comparison of the conformations of the target prolyl-residues in the enzyme-substrate complex structures of TaPHD, HsPHD2, $\mathrm{PPHD}$, and $\mathrm{CrP} 4 \mathrm{H}$.

Notes: (A) TaPHD.TaODD, (B) HsPHD2.CODD (PDB: 3HQR, ${ }^{34}$ ), (C) HsPHD2.NODD (PDB: 5L9V,,$\left.^{34}\right)$, (D) PPHD.EF-Tu, (PDB: 4IW3, ${ }^{38}$ ) and (E) CrP4H.proline-rich substrate (PDB: 3GZE, ${ }^{66}$ ) complex structures. Note that, in all the enzyme-substrate complexes, the proline C-4- methylene, that is hydroxylated, adopts the endo-conformation. (F) By contrast, Hyp564 in HsHIFI $\alpha$ CODD adopts the C-4 exo-conformation when bound to the VCB complex (PDB: ILM8, $5-57$ ). In (A and D), N-oxalylglycine (NOG) acts as a $2 \mathrm{OG}$ analog. Note that the $\mathrm{Zn}$ (substituting for $\mathrm{Fe}$ ) in the $\mathrm{CrP} 4 \mathrm{H}$ active site is tetrahedrally coordinated, with an acetate binding instead of the 2OG co-substrate. The structures reveal conserved orientations of the "target" proline residues, which in each case, adopt a C-4 endo-conformation. ${ }^{55}$ Note that the metal bound water present in the TaPHD (and HsPHD2/PPHD) substrate complex structures is not observed in the $\mathrm{CrP4H}$.(Ser-Pro) 5 structure.

ing that core features necessary for the oxygen-sensing role of the enzymes are conserved between TaPHD and HsPHD2.

\section{Comparison of ODD binding modes for TaPHD and HsPHD2}

The main substrate binding elements in the TaPHD.TaODD complex structure, as in HsPHD2, comprise: the active site containing groove, the $\beta 2 / \beta 3$-finger-loop, and the $C$-terminal $\alpha 4$-helix (Figure 3B). The TaODD peptide binds to TaPHD in an extended form; notably, the residues $N$-terminal to the target proline form a 3 -helix. The target proline in TaODD and its $N$-terminally flanking residues form a YXXLAP motif, which differs by one residue (L->Y) compared to the consensus LXXLAP prolyl-hydroxylation site(s) present in all human, and many, but not all animal, HIF $\alpha$ proteins. ${ }^{16}$ 
Binding and positioning of the hydroxylation target $\mathrm{P} 486_{\text {TaODD }}$ directly adjacent to the metal in the active site of $T a$ PHD is highly similar to that in the HsPHD2.CODD and $H s$ PHD2.NODD complex structures (Figure 4A and B). Residues Q137 ${ }_{\text {TaPHD}}, \mathrm{L}_{138_{\text {TaPHD}}}$, and A139 ${ }_{\text {TaPHD }}$ in the $\beta 2 / \beta 3$-fingerloop region (corresponding to Q239 ${ }_{H s \mathrm{PHD}}, \mathrm{L} 240_{\mathrm{HS} \mathrm{PHD}}$, and V241 ${ }_{H S \mathrm{PHD} 2}$ ) form H-bonds and hydrophobic contacts with TaODD residues $\mathrm{P} 486_{\text {TaODD, }}$ F487 $7_{\text {TaODD, }}$ A485 ${ }_{\text {TaODD }}$ (P564 ${ }_{\text {HsHF } 10}$

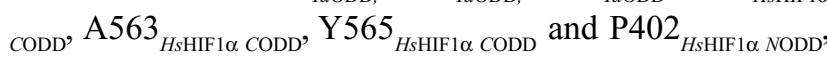
$\mathrm{A} 401_{H s \mathrm{HIF} 1 \alpha \text { NODD }}, \mathrm{A} 403_{H S H F 1 \alpha \text { NODD }}$, respectively). The hydroxylation target proline is apparently further positioned via polar interactions with $\beta \mathrm{II}(\beta 5) / \beta \mathrm{III}(\beta 6)$ residues $\mathrm{Y} 206_{\text {TaPHD }}$ and R218 $8_{T a \mathrm{PHD}}\left(\mathrm{Y} 310_{H s \mathrm{PHD} 2}\right.$ and R322 $\left.{ }_{\mathrm{Hs} \mathrm{PHD} 2}\right)$, and hydrophobic contacts are formed to residue $\mathrm{W} 285_{\text {TaPHD }}\left(\mathrm{W} 389_{\mathrm{Hs} \mathrm{PHD}}\right)$ in the $C$-terminal strand $\beta$ VIII $(\beta 11)$, (Table S1, Figure S4).

As observed in the HsPHD2.CODD/NODD structures, the substrate proline $\mathrm{P} 486_{T a O D D}$ pyrrolidine ring adopts a $\mathrm{C}-4$ endo-conformation, with the $4 R \mathrm{C}-\mathrm{H}$ bond that is cleaved during hydroxylation, being positioned close to the metal center (distance: $4.5 \AA$, Figures $3 \mathrm{C}$ and 6, ${ }^{33,34}$ ); indeed, the conformations of the substrate target prolyl rings at the TaPHD and $H_{s} \mathrm{PHD} 2$ active sites are nearly identical (Figure 4A and B). Upon hydroxylation by $H_{s} \mathrm{PHD} 2$, the conformation of the pyrrolidine ring in CODD is proposed to switch from C-4 endo to $\mathrm{C}-4$ exo $^{55}$ (Figure 6). When bound to $\mathrm{pVHL}$, the $\mathrm{C}-4$ hydroxylated proline adopts the $\mathrm{C}-4$ exo conformation as favored by stereoelectronic theory (the gauche effect); ${ }^{.55}$ a role for this conformational change in promoting product release from $H s$ PHD2 has been suggested. ${ }^{34}$

In the human PHDs, the $\beta 2 / \beta 3$-finger-loop plays a significant role in substrate recognition and in determining NODD/ CODD selectivity. ${ }^{33,34,59}$ The $\beta 2 / \beta 3$-finger-loop is partially disordered without its substrate bound, likely reflecting flexibility relative to the enzyme-substrate complex. ${ }^{33}$ By contrast, in both the HsPHD2.CODD and $H s$ PHD2.NODD structures, the $\beta 2 / \beta 3$-loop residues fold to enclose the LXXLAP substrate motif at the active site (Figure 4A and B). Although, the $\beta 2 / \beta 3$-finger-loop in the TaPHD.TaODD complex also constitutes one of the major substrate interaction sites, it is two residues shorter than in $H s$ PHD2 between N141 $1_{\text {TaPHD }}-\mathrm{V} 142_{\text {TaPHD }}$ (Figures S4 and 6). As a consequence, and by contrast to $H s \mathrm{PHD} 2,{ }^{33}$ the $\beta 2 / \beta 3$ - finger-loop in the $\mathrm{TaPHD}$.TaODD structure is more condensed and stabilized by intramolecular interactions between loop residues, including backbone-backbone H-bonds and salt bridges between side chains (Figure S3). This structural difference might reflect the fact that there is only a single HIF $\alpha$ isoform with one ODD in T. adhaerens, which contrasts with the situation in humans where there are three HIF $\alpha$ isoform substrates; with both a NODD and a CODD in case of HIF $1 \alpha$ and HIF $2 \alpha$ (note other $H s$ PHD substrates have also been reported) ${ }^{60-65}$ It is thus possible that binding of multiple HIF substrates to $H s \mathrm{PHD}_{2}{ }^{33,34}$ requires a higher degree of flexibility in the $\beta 2 /$ $\beta 3$-finger-loop, compared to the apparently more rigid $\beta 2 /$ $\beta 3$-finger-loop in TaPHD.

In both the TaPHD.TaODD and HsPHD2.CODD ${ }^{34}$ structures, a salt-bridge between an arginine residue in the $C$-terminal $\alpha 4$-helix and an aspartate residue in the $C$-terminus of the respective peptide (R292 ${ }_{\text {TaPHD }} / \mathrm{D} 494_{\text {TaODD, }} \mathrm{R} 396_{H s \mathrm{PHD} 2} /$ D571 ${ }_{H S H F 1 \alpha \text { CODD }}$ ) is formed (Figure S4). However, this saltbridge is not present in the $H s$ PHD2.NODD complex structure, where the $H s H I F 1 \alpha$ NODD peptide is positioned further from the $C$-terminal helix and interacts with the $C$-terminal region of $H_{s}$ PHD2 via hydrophobic contacts. Studies with the $T a \mathrm{ODD}$ and $H s \mathrm{PHD} 2$ variants, including with $H s$ PHD2 $\mathrm{P} 317 \mathrm{R}_{H S \mathrm{PHD} 2}$ and $\mathrm{R} 396 \mathrm{~T}_{H S \mathrm{PHD} 2}$, which are selective for CODD or NODD, respectively, support the proposal of a similar binding mode for TaODD and $H s$ HIF $1 \alpha$ CODD/NODD to HsPHD2 (Figure S4).

By contrast with $H s$ HIF $1 \alpha$ CODD/NODD peptides, the $\mathrm{TaODD}$ adopts a short $3_{10}$-helix near its Pro-Pro-Pro motif ( $\mathrm{P} 489_{\text {TaODD }}-491_{\text {TaODD }}$ ), which is $C$-terminal to the target proline $\mathrm{P} 486_{\text {TaODD }}$ residue (Figure 4A and B). Notably, the Pro-ProPro motif and the adjacent $3_{10}$-helix constitute the interaction surface with the $C$-terminal $\alpha 4$-helix of $T a \mathrm{PHD}$ (Figure 4A and B). In the TaPHD.TaODD complex structure, the TaODD is positioned particularly close to the $\alpha 4$-helix of TaPHD, compared to both $H s \mathrm{HIF} 1 \alpha$ CODD and HsPHD2.NODD, whereas the latter is positioned even more distant from the $C$-terminus of $H s$ PHD2 (Figure $4 \mathrm{~A}$ and B).

\section{Comparison of HIF- and non-HIF prolyl- 4-hydroxylase structures}

Crystal structures of the enzyme-substrate complex of a prolyl-4-hydroxylase from Chlamydomas reinhardtii $(\mathrm{Cr} \mathrm{P} 4 \mathrm{H})$, a member of the collagen-prolyl-4-hydroxylase-subfamily, ${ }^{66}$ and a Pseudomonas putida prolyl hydroxylase (PPHD), a clear prokaryotic PHD homologue, have been reported. ${ }^{38,66}$ Both CrP4H and PPHD act on non-HIF $\alpha$ substrates, ie (for consistency), $\mathrm{Cr} \mathrm{P} 4 \mathrm{H}$ catalyzes the hydroxylation of a proline residue P6 in a proline-rich (Ser-Pro) 5 substrate (PDB: $3 \mathrm{GZE},{ }^{66}$ ), and PPHD hydroxylates a proline residue (P54) in the translation elongation factor EF-Tu (PDB: 4IW3 ${ }^{38}$ ) (Figure 5).

Structure based sequence alignment of TaPHD with the human PHD isoforms, Chlamydomonas reinhardtii $(\mathrm{Cr} \mathrm{P} 4 \mathrm{H})$ and Pseudomonas putida (PPHD) was performed 
(Figure S2). Consistent with its essential role in catalysis, the 2OG-oxygenase domain $\left(\mathrm{E} 90_{T a \mathrm{PHD}}-\mathrm{F} 287_{\mathrm{Ta} \mathrm{PHD}}\right)$ manifests the highest degree of sequence conservation among the HIFand non-HIF-prolyl-4-hydroxylases. The $N$-terminal region is less well conserved, but like $H s$ PHD2, TaPHD contains a conserved cysteine-rich MYND-type zinc finger domain. The function of the $N$-terminal domain of the PHDs is not fully understood; a role for the MYND domain in the interaction with heat shock protein 90 (HSP90), co-chaperones p23, and FKBP38 has been proposed. ${ }^{67}$

Analysis of the structural conservation between TaPHD and $H s$ PHD2 (Figure S4, Table S2) reveals high overall similarity (RMSD for all $\mathrm{C} \alpha$ of $T a \mathrm{PHD}_{\mathrm{C} 73-\mathrm{Q} 297} \cdot \mathrm{TaODD}$ $H s \mathrm{PHD} 2_{\mathrm{Q} 184-\mathrm{K} 408} . \mathrm{CODD}=0.54 \AA$, and $\mathrm{RMSD}$ for all $\mathrm{C} \alpha$ of $\left.T a \mathrm{PHD}_{\mathrm{C} 73-\mathrm{Q} 297} \cdot T a \mathrm{ODD}-H_{s} \mathrm{PHD} 2_{\mathrm{P} 189-\mathrm{Y} 403} . \mathrm{NODD}=0.39 \AA\right)$. Comparisons with the other prolyl-4-hydroxylases imply that TaPHD shares a stronger structural conservation with PPHD (RMSD for all $\mathrm{C} \alpha$ of TaPHD ${ }_{\mathrm{C} 73-\mathrm{Q} 297} \cdot T a \mathrm{ODD}-\mathrm{PPHD}_{\mathrm{H} 7 \mathrm{-F} 207}$. $\mathrm{EF}-\mathrm{Tu}=0.92 \AA$ ), than with $\mathrm{CrP} 4 \mathrm{H}$ (RMSD for all $\mathrm{C} \alpha$ of $\left.T a \mathrm{PHD}_{\mathrm{C} 73-\mathrm{Q} 297} \cdot T a \mathrm{ODD}-\mathrm{CrP} 4 \mathrm{H}_{\mathrm{W} 38-\mathrm{G} 250} .(\text { Ser-Pro })_{5}=2.20 \AA\right)$, suggesting a closer evolutionary relationship between HIF hydroxylases and PPHD than to the algal prolyl4-hydroxylase $\mathrm{Cr} \mathrm{P} 4 \mathrm{H}$ (Figure 4). The structural similarity between PPHD and TaPHD is higher than between PPHD and $H_{s} \mathrm{PHD} 2$ (RMSD for all $\mathrm{C} \alpha$ of $H_{s} \mathrm{PHD} 2{ }_{\mathrm{Q} 184-\mathrm{K} 408} . \mathrm{CODD}$ - $\mathrm{PPHD}_{\mathrm{H} 7-\mathrm{F} 207} . \mathrm{EF}-\mathrm{Tu}=1.57 \AA$ ) (Figures 4 and 5).

In addition to their overall structural conservation, the prolyl-4-hydroxylases adopt strikingly similar substrate-

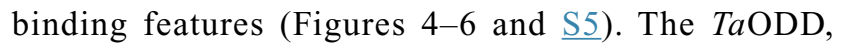
$H s$ HIF $1 \propto$ CODD/NODD, EF-Tu, and the proline-rich (Ser-Pro) 5 substrate backbones are all oriented in the same $\mathrm{N} \rightarrow \mathrm{C}$ direction across the active site (Figure $5 \mathrm{~A}$ and $\mathrm{B}$ ). $T a \mathrm{ODD}, H s \mathrm{HIF} 1 \alpha$ CODD, and $H s$ HIF $1 \alpha$ NODD all form a partial helical structure ( $3_{10}$-helix), when bound to enzyme (Figure 4A and B), while isolated $H s \mathrm{HIF} 1 \alpha$ CODD/NODD are predicted to be disordered in solution. ${ }^{33,34}$ By contrast, the EF-Tu switch loop retains loop secondary structure upon binding to PPHD, albeit after a large conformational change. ${ }^{38}$

Notably, the PHDs all employ a flexible $\beta 2 / \beta 3$-finger-loop $\left(\mathrm{G} 136_{T a \mathrm{PHD}}-\mathrm{D} 150_{T a \mathrm{PHD}}, \mathrm{S} 77_{C r \mathrm{P} 4 \mathrm{H}}-\mathrm{S} 95_{C r \mathrm{P} 4 \mathrm{H}}, \mathrm{A} 53_{\mathrm{PPHD}}-\mathrm{D} 70_{\mathrm{PPHD}}\right)$ that undergoes a major conformational change upon substrate binding and helps to enclose the target proline of the substrate in the active site cleft (Figures 5, 6 and $\underline{\mathrm{S} 4}$ ). ${ }^{33,34} \mathrm{In}$ TaPHD, HsPHD2 and PPHD, the $C$-terminus interacts with the substrate; it is unknown if this is the case for $\mathrm{Cr} \mathrm{P} 4 \mathrm{H}$, since the shorter peptide substrate used in the $\mathrm{Cr}$ P4H.(Ser-Pro) ${ }_{5}$ structure does not appear to reach the $C$-terminus of the enzyme. ${ }^{66}$

\section{Conservation of proline-ring conformation}

Comparison of the binding modes of the prolyl-4-hydroxylase substrates in the immediate active sites reveals a high degree of conservation (Figures 5A, B and 6). The substrates all manifest a similar orientation toward the active site residues and the metal center, and the target prolyl residues all adopt the C-4 endo-conformation (Figure 5A and B). ${ }^{55}$ Similarly to the HIF prolyl-4-hydroxylases TaPHD and $H_{s}$ PHD2, PPHD and CrP4H both employ a HXD...H motif for metalbinding in the active site $\left(\mathrm{H} 143_{C r \mathrm{P} 4 \mathrm{H}}, \mathrm{D} 145_{C r \mathrm{P} 4 \mathrm{H}}, \mathrm{H} 227_{C r \mathrm{P} 4 \mathrm{H}}\right.$, and $\left.\mathrm{H} 124_{\mathrm{PPHD}}, \mathrm{D} 126_{\mathrm{PPHD}}, \mathrm{H} 183_{\mathrm{PPHD}}\right){ }^{38,66}$ In the PPHD crystal structure, the metal ion is octahedrally coordinated and one of the coordination sites is occupied by a water molecule (Figure 6), implying evolutionary conservation in the active site arrangement between the HIF prolyl-4-hydroxylases in animals and the bacterial non-HIF prolyl-4-hydroxylase PPHD. By contrast, in the $C r \mathrm{P} 4 \mathrm{H}$ crystal structure, ${ }^{66}$ the metal ion is tetrahedrally coordinated; however, in this structure, binding of a water molecule to the metal is apparently blocked by an acetate ion (Figure 6E), and hence, at least in this detail, it may not be fully representative of the solution structure.

\section{Discussion}

It has been proposed that the ferrous iron dependent oxygenases may have evolved as a response to the advent of photosynthetically produced oxygen, possibly from precursors that used relatively bioavailable iron (comparable to eg, zinc) ions, predominantly in non-redox processes. ${ }^{68,69}$ The widespread occurrence of $2 \mathrm{OG}$ oxygenases in prokaryotes, coupled with their close relationship with two TCA cycle intermediates (2OG and succinate), suggests they may have early origins. The discovery that $2 \mathrm{OG}$ oxygenases play key roles in hypoxia sensing in higher animals was therefore of interest from a broad evolutionary perspective. We are interested in exploring the biological distribution of hypoxia sensing mechanisms, as well as $20 \mathrm{O}$ oxygenases and related enzymes. We have found that there is a functional HIF $\alpha$-PHD-pVHL triad in the simplest animal, T. adhaerens. ${ }^{16}$ Although bioinformatics and limited experimental analyses have not provided evidence for HIF transcription factors beyond animals, ${ }^{16}$ PHD-like enzymes are much more widely distributed (Figure S2). ${ }^{16,37,38}$

The PHDs are related to the pro-collagen prolyl hydroxylases and are part of a structurally distinct $2 \mathrm{OG}$ oxygenase subfamily; the PH VIII subfamily ${ }^{15}$ which includes other PHDs of biological interest. In the unicellular eukaryote Dictyostelium discoideum, a cytoplasmic PHD homologue, which is proposed to be involved in hypoxic responses, 
catalyzes hydroxylation of a proline-residue in Skp1, inducing further post-translational modification of Skp1 with a pentasaccharide group that is linked via the hydroxyproline residue alcohol. ${ }^{37,70}$ Recent studies have shown that PHD homologues also exist in bacteria, where a PHD present in Pseudomonas species catalyzes hydroxylation of the ribosome associated protein EF-Tu. ${ }^{38}$

Central to the hypoxia sensing ability of the PHDs is the capacity for their hydroxylase activity in many cell types to be limited by oxygen availability. ${ }^{12,29}$ It is proposed that this is in substantial part due to the slow reaction of oxygen with the active site $\mathrm{Fe}$ (II) of the PHDs. ${ }^{31}$ By contrast there is evidence that the human PHDs are (at least normally) relatively less sensitive to changes in $\mathrm{Fe}(\mathrm{II})$ or $2 \mathrm{OG}$ availability, consistent with the proposed specialized role for them as hypoxia sensors. ${ }^{30}$

The question then arises as to what extent are these apparently special properties of the human PHDs (especially HsPHD2) conserved in the PHDs/PHD-like enzymes present in animals and beyond. The results presented here provide evidence that the apparently unusual kinetic properties of $H s$ PHD2 are conserved in TaPHD (Figure S7), likely as a consequence of the conservation of key structural features.

The overall folds of the catalytic domains of TaPHD and the $H s$ PHD2 are very similar, including with respect to the elements involved in substrate binding, in particular the conformationally mobile $\beta 2 / \beta 3$-finger-loop ${ }^{33}$ and the $C$-terminal helix (Figures 5 and 6). Detailed comparison of the PHD-substrate interactions reveals that the active site region interactions between $\mathrm{TaPHD}$ and $\mathrm{TaODD}$ more closely resemble those between $H_{s} \mathrm{PHD} 2$ and $H_{s} \mathrm{HIF} 1 \alpha$ CODD, rather than those of $H s$ PHD 2 and $H s H I F 1 \alpha$ NODD (Figures 4 and 6). Thus, our results support the proposal that the PHD2/CODD-type ODD couple evolved first and is likely the most important of the PHD/ODD interactions in higher animals where there are multiple PHDs and ODDs. ${ }^{16}$ Given the likely relatively rigid nature of the Pro-Pro-Pro motif in $T a \mathrm{ODD}$, it also appears that $T a \mathrm{ODD}$ binds to $T a \mathrm{PHD}$ in a less flexible manner than does $H s$ HIF $1 \alpha$ NODD to $H s \mathrm{PHD} 2$, and to a lesser extent, $H s \mathrm{HIF} 1 \alpha \mathrm{CODD}$ (Figure 4). Although solution studies are required to validate this proposal, these differences may further reflect the evolved roles of the $H s$ PHDs (and likely those in other higher animals) in accepting multiple substrates (NODDs/CODDs of HIF $\alpha$ isoforms), and maybe, non-HIF substrates ${ }^{60-65}$ compared to TaPHD. The conservation in overall fold is also the case with respect to the prokaryotic PHD, PPHD, which acts on the highly abundant globular GTP-utilizing protein
EF-Tu, ${ }^{38}$ rather than the disordered ODD regions of HIF $\alpha$ (Figure 5). (A role of PPHD in hypoxia sensing, if any, has yet to be identified).

Crucially, the structural elements which enable the unusually slow reaction of $H s$ PHD2 with oxygen, ${ }^{31,34}$ (as well as tight $2 \mathrm{OG} / \mathrm{Fe}$ (II) binding and low substrate uncoupled turnover), appear to be conserved in TaPHD as observed crystallographically and manifested in the available kinetic studies (Figures 2 and 6). Since humans and T. adhaerens are at near opposite ends of animal evolution, it seems possible that hypoxia sensing PHD homologues in organisms between them will have similar kinetic properties. Interestingly, TaPHD has a higher affinity for 2OG than $H s$ PHD2 as observed by both $\mathrm{K}_{\mathrm{m}}$ comparisons and NMR binding studies. The difference in $2 \mathrm{OG} \mathrm{K}_{\mathrm{m}}$ may reflect different $2 \mathrm{OG}$ metabolism in T. adhaerens and humans. Further, like $H_{s}$ PHD2, TaPHD forms an unusually stable TaPHD.Fe.2OG complex (half-life $>24 \mathrm{~h}$ ), consistent with both enzymes being specialized to preferentially respond to changes in oxygen, rather than $2 \mathrm{OG} / \mathrm{Fe}(\mathrm{II})$, availability. ${ }^{11}$ Our overall results suggest that the evolution of the HIF $\alpha$ (with a TaODD/CODD type ODD)-PHD2-pVHL triad, may have been an important step in evolution of animals. A key element of this triad is a PHD2type enzyme with appropriate kinetic properties, enabling it to "focus" on hypoxia sensing. The results also suggest that searching for appropriate kinetic and structural properties may be a general approach in helping to identify candidate (hypoxia) sensing enzymes.

\section{Data availability}

The TaPHD.Mn and TaPHD.TaODD structures are deposited in the RCSB PBD: entries 6EY1 and 6F0W, respectively.

\section{Acknowledgments}

This work was supported by the Wellcome Trust, Biotechnology and Biological Sciences Research Council (BBSRC), the Leverhulme Trust, the National Institutes of Health (NIH), Cancer Research UK (CRUK), the Biochemical Society Krebs Memorial Award and a Junior Research Fellowship from Kellogg College Oxford (to MIA), and the Abraham Newton Award. We thank the Diamond Synchrotron staff for access and assistance; (Data were collected under proposal $\mathrm{mx} 1230$ ).

\section{Author contributions}

All authors contributed to data analysis, drafting or revising the article, gave final approval of the version to be published, and agree to be accountable for all aspects of the work. 


\section{Disclosure}

The authors report no conflicts of interest in this work.

\section{References}

1. Epstein AC, Gleadle JM, McNeill LA, et al. C. elegans EGL-9 and mammalian homologs define a family of dioxygenases that regulate HIF by prolyl hydroxylation. Cell. 2001;107(1):43-54.

2. Kaelin WG. Proline hydroxylation and gene expression. Annu Rev Biochem. 2005;74:115-128.

3. Kaelin WG, Ratcliffe PJ. Oxygen sensing by metazoans: the central role of the HIF hydroxylase pathway. Mol Cell. 2008;30(4):393-402.

4. Schofield CJ, Ratcliffe PJ. Oxygen sensing by HIF hydroxylases. Nat Rev Mol Cell Biol. 2004;5(5):343-354.

5. Chowdhury R, Hardy A, Schofield CJ. The human oxygen sensing machinery and its manipulation. Chem Soc Rev. 2008;37(7):1308-1319.

6. Semenza GL. Oxygen-dependent regulation of mitochondrial respiration by hypoxia-inducible factor 1. Biochem J. 2007;405(1):1-9.

7. Ivan M, Kondo K, Yang H, et al. HIF $\alpha$ targeted for VHL-mediated destruction by proline hydroxylation: implications for $\mathrm{O}_{2}$ sensing. Science. 2001;292(5516):464-468.

8. Jaakkola P, Mole DR, Tian YM, et al. Targeting of HIF- $\alpha$ to the von Hippel-Lindau ubiquitylation complex by $\mathrm{O}_{2}$-regulated prolyl hydroxylation. Science. 2001;292(5516):468-472.

9. Maxwell PH, Wiesener MS, Chang GW, et al. The tumour suppressor protein VHL targets hypoxia-inducible factors for oxygen-dependent proteolysis. Nature. 1999;399(6733):271-275.

10. Hewitson KS, McNeill LA, Riordan MV, et al. Hypoxia-inducible factor (HIF) asparagine hydroxylase is identical to factor inhibiting HIF (FIH) and is related to the cupin structural family. J Biol Chem. 2002;277(29):26351-26355.

11. Lando D, Peet DJ, Whelan DA, Gorman JJ, Whitelaw ML. Asparagine hydroxylation of the HIF transactivation domain a hypoxic switch. Science. 2002;295(5556):858-861.

12. Tian YM, Yeoh KK, Lee MK, et al. Differential sensitivity of hypoxia inducible factor hydroxylation sites to hypoxia and hydroxylase inhibitors. J Biol Chem. 2011;286(15):13041-13051.

13. Koivunen P, Hirsilä M, Günzler V, Kivirikko KI, Myllyharju J. Catalytic properties of the asparaginyl hydroxylase $(\mathrm{FIH})$ in the oxygen sensing pathway are distinct from those of its prolyl 4-hydroxylases. $J$ Biol Chem. 2004;279(11):9899-9904.

14. Elkins JM, Hewitson KS, McNeill LA, et al. Structure of factor-inhibiting hypoxia-inducible factor (HIF) reveals mechanism of oxidative modification of HIF-1 $\alpha$. J Biol Chem. 2003;278(3):1802-1806.

15. Aik WS, Chowdhury R, Clifton IJ, et al. Introduction to structural studies on 2-oxoglutarate-dependent oxygenases and related enzymes. In: Hausinger RP, Schofield CJ, editors. 2-Oxoglutarate-Dependent Oxygenases. 59-94. London: Royal Society of Chemistry; 2015: $59-94$.

16. Loenarz C, Coleman ML, Boleininger A, et al. The hypoxia-inducible transcription factor pathway regulates oxygen sensing in the simplest animal, Trichoplax adhaerens. EMBO Rep. 2011;12(1):63-70.

17. Chan MC, Holt-Martyn JP, Schofield CJ, Ratcliffe PJ. Pharmacological targeting of the HIF hydroxylases - a new field in medicine development. Mol Aspects Med. 2016;47-48:54-75.

18. Yan L, Colandrea VJ, Hale JJ. Prolyl hydroxylase domain-containing protein inhibitors as stabilizers of hypoxia-inducible factor: small molecule-based therapeutics for anemia. Expert Opin Ther Pat. 2010;20(9):1219-1245.

19. Berra E, Benizri E, Ginouvès A, Volmat V, Roux D, Pouysségur J. HIF prolyl-hydroxylase 2 is the key oxygen sensor setting low steady-state levels of HIF-1 $\alpha$ in normoxia. EMBO J. 2003;22(16):4082-4090.

20. Takeda K, Ho VC, Takeda H, Duan LJ, Nagy A, Fong GH. Placental but not heart defects are associated with elevated hypoxia-inducible factor $\alpha$ levels in mice lacking prolyl hydroxylase domain protein 2. Mol Cell Biol. 2006;26(22):8336-8346.
21. Hirsilä M, Koivunen P, Günzler V, Kivirikko KI, Myllyharju J. Characterization of the human prolyl 4-hydroxylases that modify the hypoxiainducible factor. $J$ Biol Chem. 2003;278(33):30772-30780.

22. Huang J, Zhao Q, Mooney SM, Lee FS. Sequence determinants in hypoxia-inducible factor- $1 \alpha$ for hydroxylation by the prolyl hydroxylases PHD1, PHD2, and PHD3. J Biol Chem. 2002;277(42):39792-39800.

23. Chan DA, Sutphin PD, Yen SE, Giaccia AJ. Coordinate regulation of the oxygen-dependent degradation domains of hypoxia-inducible factor

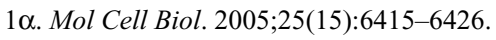

24. Masson N, Willam C, Maxwell PH, Pugh CW, Ratcliffe PJ. Independent function of two destruction domains in hypoxia-inducible factor- $\alpha$ chains activated by prolyl hydroxylation. EMBO J. 2001;20(18): 5197-5206.

25. Heikkilä M, Pasanen A, Kivirikko KI, Myllyharju J. Roles of the human hypoxia-inducible factor (HIF)-3 $\alpha$ variants in the hypoxia response. Cell Mol Life Sci. 2011;68(23):3885-3901.

26. Makino Y, Cao R, Svensson K, et al. Inhibitory PAS domain protein is a negative regulator of hypoxia-inducible gene expression. Nature. 2001;414(6863):550-554.

27. Yamashita T, Ohneda O, Nagano M, et al. Abnormal heart development and lung remodeling in mice lacking the hypoxia-inducible factorrelated basic helix-loop-helix PAS protein NEPAS. Mol Cell Biol. 2008;28(4):1285-1297.

28. Ehrismann D, Flashman E, Genn DN, et al. Studies on the activity of the hypoxia-inducible-factor hydroxylases using an oxygen consumption assay. Biochem J. 2007;401(1):227-234.

29. Tarhonskaya H, Chowdhury R, Leung IK, et al. Investigating the contribution of the active site environment to the slow reaction of hypoxiainducible factor prolyl hydroxylase domain 2 with oxygen. Biochem $J$. 2014;463(3):363-372.

30. McNeill LA, Flashman E, Buck MR, et al. Hypoxia-inducible factor prolyl hydroxylase 2 has a high affinity for ferrous iron and 2-oxoglutarate. Mol Biosyst. 2005;1(4):321-324.

31. Flashman E, Hoffart LM, Hamed RB, Bollinger JM, Krebs C, Schofield CJ. Evidence for the slow reaction of hypoxia-inducible factor prolyl hydroxylase 2 with oxygen. FEBS J. 2010;277(19):4089-4099.

32. McDonough MA, Li V, Flashman E, et al. Cellular oxygen sensing: Crystal structure of hypoxia-inducible factor prolyl hydroxylase (PHD2). Proc Natl Acad Sci U S A. 2006;103(26):9814-9819.

33. Chowdhury R, Leung IK, Tian YM, et al. Structural basis for oxygen degradation domain selectivity of the HIF prolyl hydroxylases. Nat Commun. 2016;7:12673.

34. Chowdhury R, McDonough MA, Mecinović J, et al. Structural basis for binding of hypoxia-inducible factor to the oxygen-sensing prolyl hydroxylases. Structure. 2009;17(7):981-989.

35. Flashman E, Hoffart LM, Hamed RB, Bollinger JM, Krebs C, Schofield CJ. Evidence for the slow reaction of hypoxia-inducible factor prolyl hydroxylase 2 with oxygen. FEBS J. 2010;277(19): 4089-4099.

36. Tarhonskaya H, Chowdhury R, Leung IK, et al. Investigating the contribution of the active site environment to the slow reaction of hypoxiainducible factor prolyl hydroxylase domain 2 with oxygen. Biochem $J$. 2014;463(3):363-372.

37. van der Wel H, Ercan A, West CM. The Skp1 prolyl hydroxylase from Dictyostelium is related to the hypoxia-inducible factor- $\alpha$ class of animal prolyl 4-hydroxylases. J Biol Chem. 2005;280(15): $14645-14655$.

38. Scotti JS, Leung IK, Ge W, et al. Human oxygen sensing may have origins in prokaryotic elongation factor Tu prolyl-hydroxylation. Proc Natl Acad Sci U S A. 2014;111(37):13331-13336.

39. Xu Y, Brown KM, Wang ZA, et al. The Skp1 protein from Toxoplasma is modified by a cytoplasmic prolyl 4-hydroxylase associated with oxygen sensing in the social amoeba Dictyostelium. J Biol Chem. 2012;287(30):25098-25110.

40. Myllyharju J. Prolyl 4-hydroxylases, key enzymes in the synthesis of collagens and regulation of the response to hypoxia, and their roles as treatment targets. Ann Med. 2008;40(6):402-417. 
41. Myllyharju J. Intracellular post-translational modifications of collagens. In: Brinckmann J, Notbohm H, Müller PK, editors. Collagen: Primer in Structure, Processing and Assembly. 115-147 Heidelberg: SpringerVerlag Berlin Heidelberg; 2005:115-147.

42. Kukkola L, Hieta R, Kivirikko KI, Myllyharju J. Identification and characterization of a third human, rat, and mouse collagen prolyl 4-hydroxylase isoenzyme. J Biol Chem. 2003;278(48):47685-47693.

43. Myllyharju J, Kivirikko KI. Characterization of the iron- and 2-oxoglutarate-binding sites of human prolyl 4-hydroxylase. EMBO J. 1997;16(6):1173-1180.

44. Nokelainen M, Nissi R, Kukkola L, Helaakoski T, Myllyharju J. Characterization of the human and mouse genes for the $\alpha$ subunit of type II prolyl 4-hydroxylase Eur J Biochem. 2001;268:5300-5309.

45. Hirsilä M, Koivunen P, Xu L, Seeley T, Kivirikko KI, Myllyharju J. Effect of desferrioxamine and metals on the hydroxylases in the oxygen sensing pathway. FASEB J. 2005;19(10):1308-1310.

46. Koivunen P, Hirsilä M, Kivirikko KI, Myllyharju J. The length of peptide substrates has a marked effect on hydroxylation by the hypoxia-inducible factor prolyl 4-hydroxylases. J Biol Chem. 2006;281(39):28712-28720.

47. Appelhoff RJ, Tian YM, Raval RR, et al. Differential function of the prolyl hydroxylases PHD1, PHD2, and PHD3 in the regulation of hypoxia-inducible factor. J Biol Chem. 2004;279(37):38458-38465

48. Hausinger RP. Fe ${ }^{\mathrm{II}} / \alpha$-ketoglutarate-dependent hydroxylases and related enzymes. Crit Rev Biochem Mol Biol. 2004;39(1):21-68.

49. Abboud MI, McAllister TE, Leung IKH, et al. 2-Oxoglutarate regulates binding of hydroxylated hypoxia-inducible factor to prolyl hydroxylase domain 2. Chemical Commun. 2018; 54(25):3130-3133.

50. Dao JH, Kurzeja RJ, Morachis JM, et al. Kinetic characterization and identification of a novel inhibitor of hypoxia-inducible factor prolyl hydroxylase 2 using a time-resolved fluorescence resonance energy transfer-based assay technology. Anal Biochem. 2009;384(2):213-223.

51. Hangasky JA, Taabazuing CY, Martin CB, Eron SJ, Knapp MJ. The facial triad in the $\alpha$-ketoglutarate dependent oxygenase $\mathrm{FIH}$ : A role for sterics in linking substrate binding to $\mathrm{O}_{2}$ activation. J Inorg Biochem. 2017;166:26-33.

52. Wilkins SE, Abboud MI, Hancock RL, Schofield CJ. Targeting proteinprotein interactions in the HIF system. ChemMedChem. 2016;11(8): 773-786

53. Neidig ML, Brown CD, Light KM, et al. CD and MCD of CytC3 and taurine dioxygenase: role of the facial triad in $\alpha$-KG-dependent oxygenases. J Am Chem Soc. 2007;129(46):14224-14231.

54. Tarhonskaya H, Hardy AP, Howe EA, et al. Kinetic Investigations of the Role of Factor Inhibiting Hypoxia-inducible Factor (FIH) as an Oxygen Sensor. J Biol Chem. 2015;290(32):19726-19742.

55. Loenarz C, Mecinović J, Chowdhury R, McNeill LA, Flashman E, Schofield CJ. Evidence for a stereoelectronic effect in human oxygen sensing. Angew Chem Int Ed Engl. 2009;48(10):1784-1787.

56. Min JH, Yang H, Ivan M, Gertler F, Kaelin WG, Pavletich NP. Structure of an HIF-1 $\alpha$-pVHL complex: hydroxyproline recognition in signaling. Science. 2002;296(5574):1886-1889.
57. Hon WC, Wilson MI, Harlos K, et al. Structural basis for the recognition of hydroxyproline in HIF-1 $\alpha$ by pVHL. Nature. 2002;417(6892): 975-978.

58. Domene C, Jorgensen C, Vanommeslaeghe K, Schofield CJ, Mackerell A. Quantifying the Binding Interaction between the Hypoxia-Inducible Transcription Factor and the von Hippel-Lindau Suppressor. J Chem Theory Comput. 2015;11(8):3946-3954.

59. Flashman E, Bagg EA, Chowdhury R, et al. Kinetic rationale for selectivity toward $N$ - and $C$-terminal oxygen-dependent degradation domain substrates mediated by a loop region of hypoxia-inducible factor prolyl hydroxylases. J Biol Chem. 2008;283(7):3808-3815.

60. Place TL, Domann FE. Prolyl-hydroxylase 3: evolving roles for an ancient signaling protein. Hypoxia. 2013;2013(1):13-27.

61. Kuznetsova AV, Meller J, Schnell PO, et al. von Hippel-Lindau protein binds hyperphosphorylated large subunit of RNA polymerase II through a proline hydroxylation motif and targets it for ubiquitination. Proc Natl Acad Sci U S A. 2003;100(5):2706-2711.

62. Luo W, Hu H, Chang R, et al. Pyruvate kinase M2 is a PHD3-stimulated coactivator for hypoxia-inducible factor 1. Cell. 2011;145(5) $732-744$.

63. Xie L, Xiao K, Whalen EJ, et al. Oxygen-Regulated $\beta(2)$-Adrenergic Receptor Hydroxylation by EGLN3 and Ubiquitylation by pVHL. Science Signal. 2009;2(78):ra33.

64. Anderson K, Nordquist KA, Gao X, et al. Regulation of cellular levels of Sprouty2 protein by prolyl hydroxylase domain and von Hippel-Lindau proteins. J Biol Chem. 2011;286(49):42027-42036.

65. Guo J, Chakraborty AA, Liu P, et al. pVHL suppresses kinase activity of Akt in a proline-hydroxylation-dependent manner. Science. 2016;353(6302):929-932.

66. Koski MK, Hieta R, Hirsilä M, Rönkä A, Myllyharju J, Wierenga RK. The crystal structure of an algal prolyl 4-hydroxylase complexed with a proline-rich peptide reveals a novel buried tripeptide binding motif. J Biol Chem. 2009;284(37):25290-25301.

67. Song D, Li LS, Heaton-Johnson KJ, Arsenault PR, Master SR, Lee FS. Prolyl hydroxylase domain protein 2 (PHD2) binds a Pro-Xaa-Leu-Glu motif, linking it to the heat shock protein 90 pathway. J Biol Chem. 2013;288(14):9662-9674.

68. Taylor CT, Mcelwain JC. Ancient atmospheres and the evolution of oxygen sensing via the hypoxia-inducible factor in metazoans. Physiology. 2010;25(5):272-279.

69. Barlow JN, Baldwin JE, Clifton IJ, et al. Studies on non-haem ferrous-dependent oxygenases and oxidases. Biochem Soc Trans. 1997;25(1):86-90.

70. West CM, Wang ZA, van der Wel H. A cytoplasmic prolyl hydroxylation and glycosylation pathway modifies Skp1 and regulates $\mathrm{O}_{2}$-dependent development in Dictyostelium. Biochim Biophys Acta. 2010;1800(2):160-171.
Hypoxia

\section{Publish your work in this journal}

Hypoxia is an international, peer-reviewed, open access journal that aims to improve understanding of the biological response to hypoxia. The journal will publish original research articles, reviews, methodological advances, clinical studies, and expert opinions that identify developments in the regulation of the physiological and pathological responses to
Dovepress

hypoxia and in the therapeutic targeting of hypoxia-responsive pathways. The manuscript management system is completely online and includes a very quick and fair peer-review system, which is all easy to use. Visit http://www.dovepress.com/testimonials.php to read real quotes from published authors. 